\title{
Metabolic reprogramming of fibro/adipogenic progenitors facilitates muscle regeneration
}

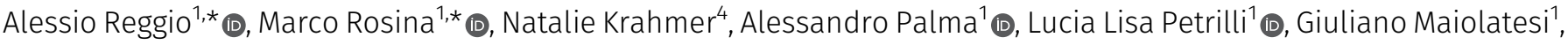

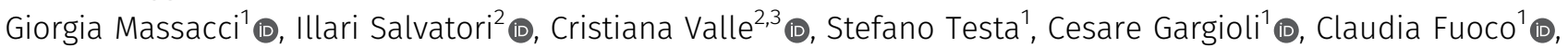

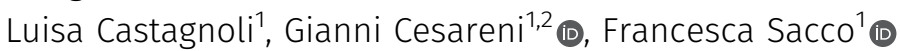

In Duchenne muscular dystrophy (DMD), the absence of the dystrophin protein causes a variety of poorly understood secondary effects. Notably, muscle fibers of dystrophic individuals are characterized by mitochondrial dysfunctions, as revealed by a reduced ATP production rate and by defective oxidative phosphorylation. Here, we show that in a mouse model of DMD ( $m d x)$, fibro/ adipogenic progenitors (FAPs) are characterized by a dysfunctional mitochondrial metabolism which correlates with increased adipogenic potential. Using high-sensitivity mass spectrometrybased proteomics, we report that a short-term high-fat diet (HFD) reprograms dystrophic FAP metabolism in vivo. By combining our proteomic dataset with a literature-derived signaling network, we revealed that HFD modulates the $\beta$-catenin-follistatin axis. These changes are accompanied by significant amelioration of the histological phenotype in dystrophic mice. Transplantation of purified FAPs from HFD-fed mice into the muscles of dystrophic recipients demonstrates that modulation of FAP metabolism can be functional to ameliorate the dystrophic phenotype. Our study supports metabolic reprogramming of muscle interstitial progenitor cells as a novel approach to alleviate some of the adverse outcomes of DMD.

DOI 10.26508/lsa.202000660 | Received 27 January 2020 | Accepted 28 January 2020 | Published online 04 February 2020

\section{Introduction}

The interaction between the dystrophin protein and the dystrophinassociated protein complex (DAPC), which spans the sarcolemmal membrane, is essential for the integrity of the muscle fibers (Petrof et $\mathrm{al}$, 1993). Dystrophin deficiency and the ensuing decrease of the DAPC components in Duchenne muscular dystrophy (DMD) patients (Emery, 1998) dramatically increases myofiber fragility upon muscle contraction and affects calcium and sodium homeostasis (Ohlendieck \& Campbell, 1991; Petrof et al, 1993). Over time, chronic muscle damage culminates in the failure of the regeneration process leading to patient paralysis and, finally, death (Emery, 1998).

In addition, dystrophin deficiency also causes a variety of poorly understood secondary effects, mostly related to mitochondrial dysfunctions in myofibers. Muscles of DMD patients and animal models have a reduced oxygen consumption, spare capacity, and mitochondrial complex I activity (Percival et al, 2012; Schuh et al, 2012; Rybalka et al, 2014). Consistently, mitochondrial enzymes of the tricarboxylic acid cycle (TCA) (Lindsay et al, 2018) and of the electron transport chain (Rybalka et al, 2014) are also significantly decreased. These functional defects correlate with mitochondrial structural abnormalities. Dense and dilated mitochondria with altered cristae as well as swollen mitochondria have been described in muscle fibers of DMD patients (Rybalka et al, 2014).

Altogether, these and additional evidences implicate a metabolic impairment in the dystrophic disease development and progression (Rodriguez-Cruz et al, 2015). Whether a causal link between the metabolic alterations and the pathological phenotype exists remains to be established.

To counteract these metabolic alterations, different nutritional approaches have been proposed, with the aim of restoring mitochondrial functionality and muscle regeneration. A reduced caloric intake or a periodic fasting-mimicking diet were shown to stimulate regeneration of different organs, including skeletal muscle, in humans and mice (Civitarese et al, 2007; Cerletti et al, 2012; Brandhorst et al, 2015). A short-term caloric restriction enhances muscle satellite cells (MuSCs) functionality, promoting muscle regeneration upon acute muscle injury in mice (Cerletti et al, 2012). At the molecular level, the AMPK-SIRT1-PGC- $1 \alpha$ axis plays a crucial role in mediating the diet-dependent increase of muscle regeneration. Consistently, pharmacological activation of AMPK by sirtuin1, resveratrol, metformin, or AICAR was shown to mitigate the dystrophic phenotype in the $m d x$ mouse model of DMD (Pauly et al, 2012; Ljubicic \& Jasmin, 2015; Hafner et al, 2016; Juban et al, 2018). A

\footnotetext{
${ }^{1}$ Department of Biology, University of Rome Tor Vergata, Rome, Italy ${ }^{2}$ Fondazione Santa Lucia Istituto di Ricovero e Cura a Carattere Scientifico (IRCCS), Rome, Italy ${ }^{3}$ Institute of Translational Pharmacology, Consiglio Nazionale delle Ricerche (CNR), Rome, Italy ${ }^{4}$ Department Proteomics and Signal Transduction, Max-Planck Institute of Biochemistry, Martinsried, Germany
}

Correspondence: francesca.sacco@uniroma2.it; cesareni@uniroma2.it; castagnoli@uniroma2.it

*Alessio Reggio and Marco Rosina contributed equally to this work 
fat-enriched diet regimen was also considered as a life-style strategy to revert the metabolic impairment of DMD. Dystrophic mice fed for 16-wk with a high-fat diet (HFD) achieved an increased running ability accompanied by a reduction of myofiber necrosis without significant weight gain (Radley-Crabb et al, 2011). In addition, a variety of nutritional approaches based on amino acid supplementation have also been shown to have beneficial effects on muscle regeneration in dystrophic mouse models (Passaquin et al, 2002; Voisin et al, 2005; Barker et al, 2017; Banfi et al, 2018). Such positive effects suggest an impact of muscle metabolism and muscle homeostasis and physiology.

The skeletal muscle is a heterogeneous tissue and its regeneration after acute or chronic damage is governed by a complex interplay between muscle-resident and circulating cell populations that in concert contribute to damage resolution (Arnold et al, 2007; Christov et al, 2007; Dellavalle et al, 2011; Murphy et al, 2011).

MuSCs are the main stem progenitor cells directly responsible for the formation of new myofibers (Seale et al, 2004; Lepper et al, 2011; Sambasivan et al, 2011). However, fibro/adipogenic progenitors (FAPS), a muscle-resident interstitial stem cell population of mesenchymal origin (Vallecillo Garcia et al, 2017), are also involved in muscle regeneration (Murphy et al, 2011). FAPs play a doubleedged role. In healthy conditions, they promote muscle regeneration by establishing crucial trophic interactions with MuSCs (Joe et al, 2010; Uezumi et al, 2010; Murphy et al, 2011), whereas in the late stages of the dystrophic pathology, they differentiate into fibroblasts and adipocytes. As a result, fibrotic scars and fat infiltrates compromise muscle structure and function (Uezumi et al, 2011). We considered whether any of these progenitor cell types, similarly to myofibers, have an altered metabolism that affects their function in dystrophic patients.

We have recently applied high-resolution mass spectrometry (MS)-based proteomics to characterize the changes in the FAP proteome upon acute (cardiotoxin) or chronic injury (Marinkovic et al, 2019). This unbiased strategy revealed that FAPs from $m d x$ mice are also characterized by a significant reduction of mitochondrial metabolic enzymes, accompanied by an increased expression of glycolytic proteins (Marinkovic et al, 2019). Here, we demonstrate that the impaired mitochondrial metabolism of dystrophic FAPs correlates with their ability to proliferate and differentiate into adipocytes. Remarkably, in vitro metabolic reprogramming of dystrophic FAPs modulates their adipogenic potential.

As lipid-rich diets have a positive effect on the DMD phenotype, we investigated the effects of in vivo metabolic reprogramming on dystrophic FAP and MuSC biology. By applying an unbiased MSbased proteomic approach, here we show that HFD not only restores mitochondrial functionality in FAPs from dystrophic mice but also rewires key signaling networks and protein complexes. Our study reveals an unexpected connection between FAP metabolic reprogramming and their ability to promote the myogenic potential of MuSCs. The integration of our proteome-wide analysis with a literature-derived signaling network identifies $\beta$-catenin as a crucial regulator of the expression of the promyogenic factor follistatin. In summary, our study reveals that in vivo metabolic reprogramming of $m d x$ FAPs correlates with a significant amelioration of the dystrophic phenotype, endorsing nutritional intervention as a promising supportive approach in the treatment of muscular dystrophies.

\section{Results}

\section{FAPs and MuSCs from dystrophic muscles have mitochondrial dysfunction and mainly rely on glycolysis to generate ATP}

Recently, we have applied MS-based proteomic approach to elucidate the mechanisms underlying the different sensitivity of dystrophic FAPs to the Neurogenic locus notch homolog protein (NOTCH)-dependent adipogenesis (Marinkovic et al, 2019). Here, we dissected the proteomic dataset focusing on the expression levels of key metabolic enzymes. We found that most of the key enzymes involved in fatty acid metabolism, TCA cycle, and oxidative phosphorylation (OxPhos) are significantly down-regulated in dystrophic as compared with wild-type (wt) FAPs (Fig 1A and B). Conversely, many of the enzymes of the glycolytic and the pentose phosphate pathways are up-regulated (Fig 1 A and B), suggesting an increased anabolic metabolism in $m d x$ FAPs. To confirm and extend these observations, we collected highly pure preparations of $\mathrm{Ly}_{6 \mathrm{~A}^{+}}\left(\mathrm{Sca} 1^{+}\right)$ FAPs and ITGA7 $7^{+}$MuSCs from wild-type (wt) and dystrophic ( $m d x$ ) mice, via magnetic bead cell sorting (Marinkovic et al, 2019; Reggio et al, 2019b). Sca1 ${ }^{+}$FAPs and ITGA7 ${ }^{+}$MuSCs express their distinctive markers PDGFR $\alpha$ and Pax7, respectively (Fig S1A-C).

To validate the conclusions drawn from the unbiased mass spectrometry dataset, we monitored the level of crucial metabolic enzymes. The key glycolytic and pro-anabolic enzyme pyruvate kinase M2 (PKM2) (Mazurek, 2011) is significantly increased in dystrophic FAPs (approximately fivefold) (Fig 1C and D) and to a lesser extent in dystrophic MuSCs (Fig 1E and F). In parallel, we also observed a significant reduction of mitochondrial complex V and III subunits in FAPs (Fig 1C and D) but not in MuSCs (Fig $1 \mathrm{E}$ and F).

To confirm that $m d x$ progenitor cells have an altered energy metabolism, we purified FAPs and MuSCs and measured the oxygen consumption rate (OCR) under mitochondrial stress test conditions (Figs 2A-J and S2A-J). In comparison with wt, the OCR of dystrophic cells in basal conditions was reduced in mdx FAPs (Fig 2B). A similar, albeit smaller, decrease was observed also in mdx MuSCs (Fig S2B). This is in accordance with the proteomic analysis, where FAPs showed a decreased concentration of enzymes in the oxidative phosphorylation pathway (Fig 1 A and B). Upon sequential injection of inhibitors of mitochondrial functions, dystrophic FAPs display a reduced mitochondrial ATP production and spare respiratory capacity, indicating that the mitochondrial efficiency and responsiveness to different energy demands are impaired in these cells (Fig $2 \mathrm{C}$ and D). By using the OCR/ extracellular acidification rate ratio as a proxy of metabolic imbalance (Pala et al, 2018), we also conclude that $m d x$ FAPs have a more robust glycolytic flux than wt matched cells (Fig 2E). Although less evident in comparison with mdx FAPs, also dystrophic MuSCs have a reduced mitochondrial performance (Fig S2C and D) and mainly exploit glycolysis for energy production (Fig S2E). Moreover, dystrophic FAPS show a lower mitochondrial trans-membrane potential and a weaker 
A

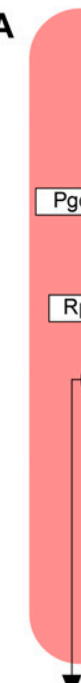

Nucleotide

Biosynthesis

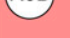

Pentose phosphate

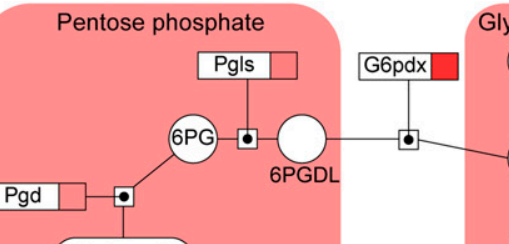

Ribulose-5-P

\begin{tabular}{|l|l|}
\hline Rpia & Rpe \\
\hline
\end{tabular}

(R5B

S7P Tkt

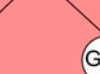
Taldo
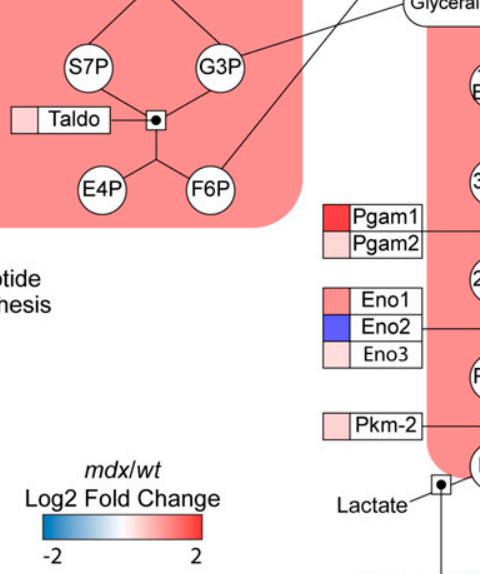

Electron tra
Complex I \begin{tabular}{|l|l|}
\hline Ndufa2 & Ndufs6 \\
\hline Ndufa3 & Ndufa5 \\
\hline
\end{tabular} Ndufa3 Ndufa

\begin{tabular}{|l|l|}
\hline Ndufa6 & Ndufs4 \\
\hline
\end{tabular}

\begin{tabular}{|l|l|}
\hline Ndufa6 & Ndufs4 \\
\hline Ndufa7 & Ndufa8 \\
\hline
\end{tabular}

Ndufb4 Ndufs8 1 Uqcrc1

Uqcrc2

Ndufa12Ndufs3 3 Uacrcb

Ndufb7 Ndufa9

Ndufb9Ndufa10 Uqcrh

Ndufa4 Ndufv1 1 Uqcr10

Ndufb10Ndufs 1

NdufabiNdufs2

Ndufv3
Ndufc2 Ndufs7 Complex II
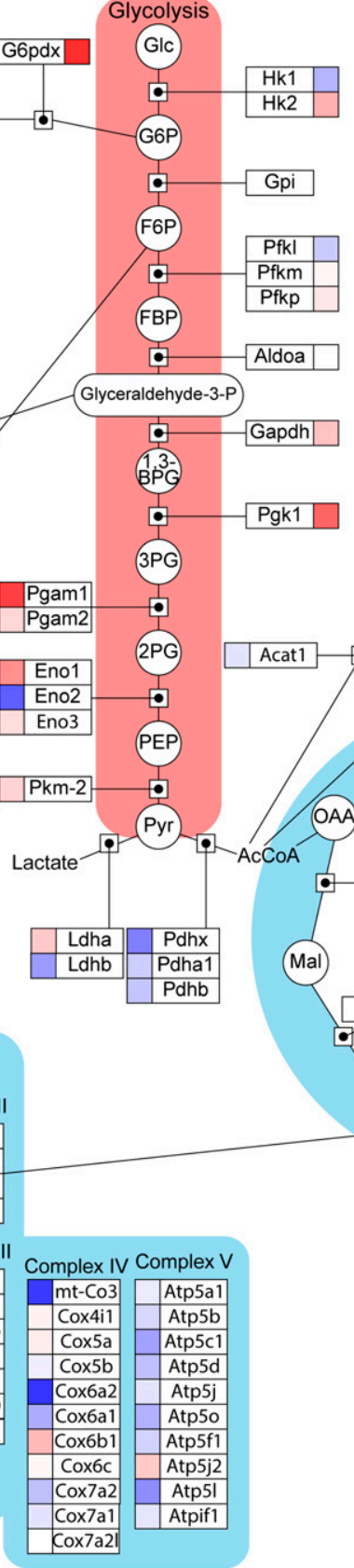

$\beta$-oxidatio

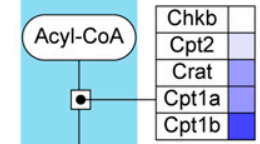

.
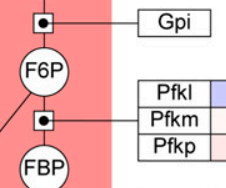

西

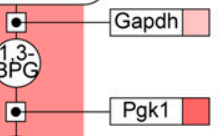

\section{.}
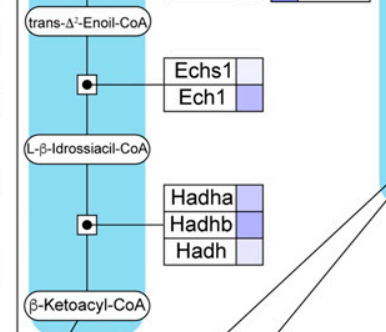

Ketoacyl-CoA

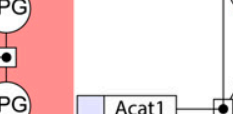

.
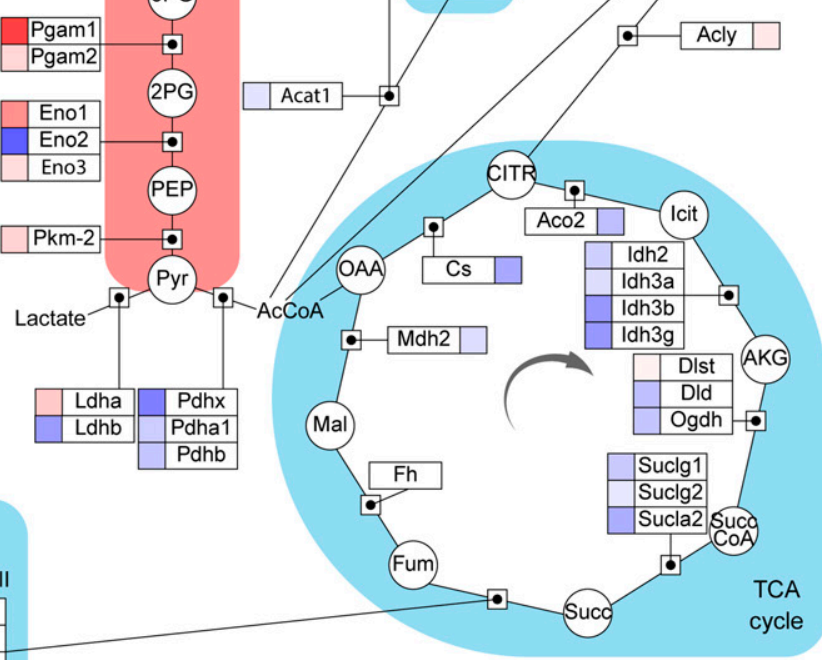

(2)

(n)

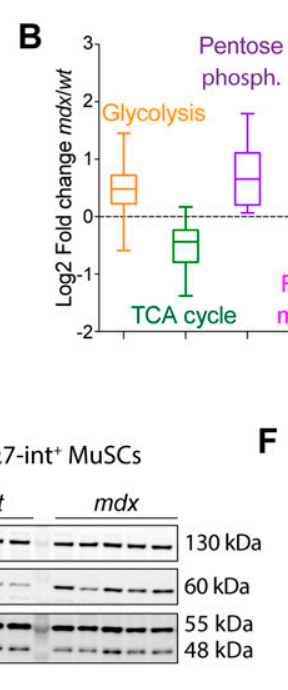

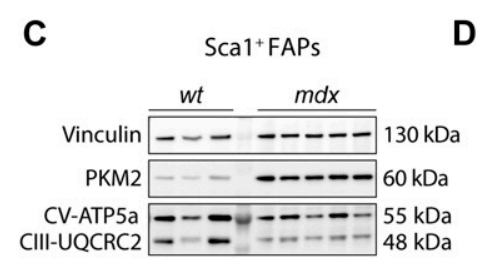

E

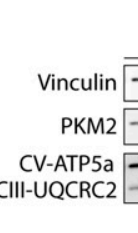

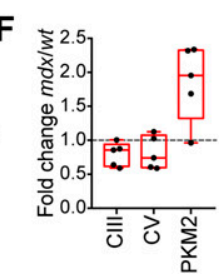

Figure 1. Mass spectrometry-based proteomics of $m d x$ fibro/ adipogenic progenitors (FAPs) reveals a significant alteration of key metabolic pathways.

(A) The metabolic pathway map derived from Wikipathways (http:// www.wikipathways.org) of key metabolic enzymes significantly modulated in mdx FAPs compared with wt. For each detected enzyme, a corresponding square is color coded according to the log2 fold change of the protein expression level in $m d x$ compared with wt FAPs. (B) Boxplot representing the log2 fold change ( $m d x / w t)$ of the abundance of metabolic enzymes annotated with the GO terms glycolysis, TCA cycle, pentose phosphate, and fatty acid metabolism in FAPs. (c) Western Blot of PKM2, CV-ATP5a, CIII-UQCRC2, and vinculin in FAPs isolated from the hind limbs of young (45-d old) wt and $m d x$ mice ( $w t$ FAPs $n=3$; $m d x$ FAPs $n=5$ ).

(D) Bar graphs representing the fold change of the enzymes PKM2, CVATP5a, and CIII-UQCRC2 in FAPS. Protein levels were normalized to vinculin. (E) Western Blot of PKM2, CV-ATP5a, CIII-UQCRC2, and vinculin in muscle satellite cells (MuSCs) isolated from the hind limbs of wt and $m d x$ mice (wt MuSCs $n=3$; $\operatorname{mdx}$ MuSCs $n=5$ ). (F) Bar graphs representing the fold change of the enzymes PKM2, CV-ATP5a, and CIII-UQCRC2 in MuSCS. Protein levels were normalized to vinculin. Statistical significance was estimated by $t$ test. All data are represented as mean \pm SEM and statistical significance is defined as ${ }^{*} P<0.05 ;{ }^{* *} P$ $<0.01 ;{ }^{* * *} P<0.001$. response to FCCP uncoupling treatment, as shown by the MitoTracker Red labelling (Fig 2K). Such reduction is not related to the change in total mitochondrial mass as shown in MitoTracker Green labelling (Fig 2L).
Our data are consistent with wt and dystrophic progenitor cells being in a different metabolic state. Whereas wt cells, similarly to other quiescent stem progenitors, mainly rely on OxPhos for energy production (Ryall et al, 2015; Knobloch et al, 2017), mdx progenitors 

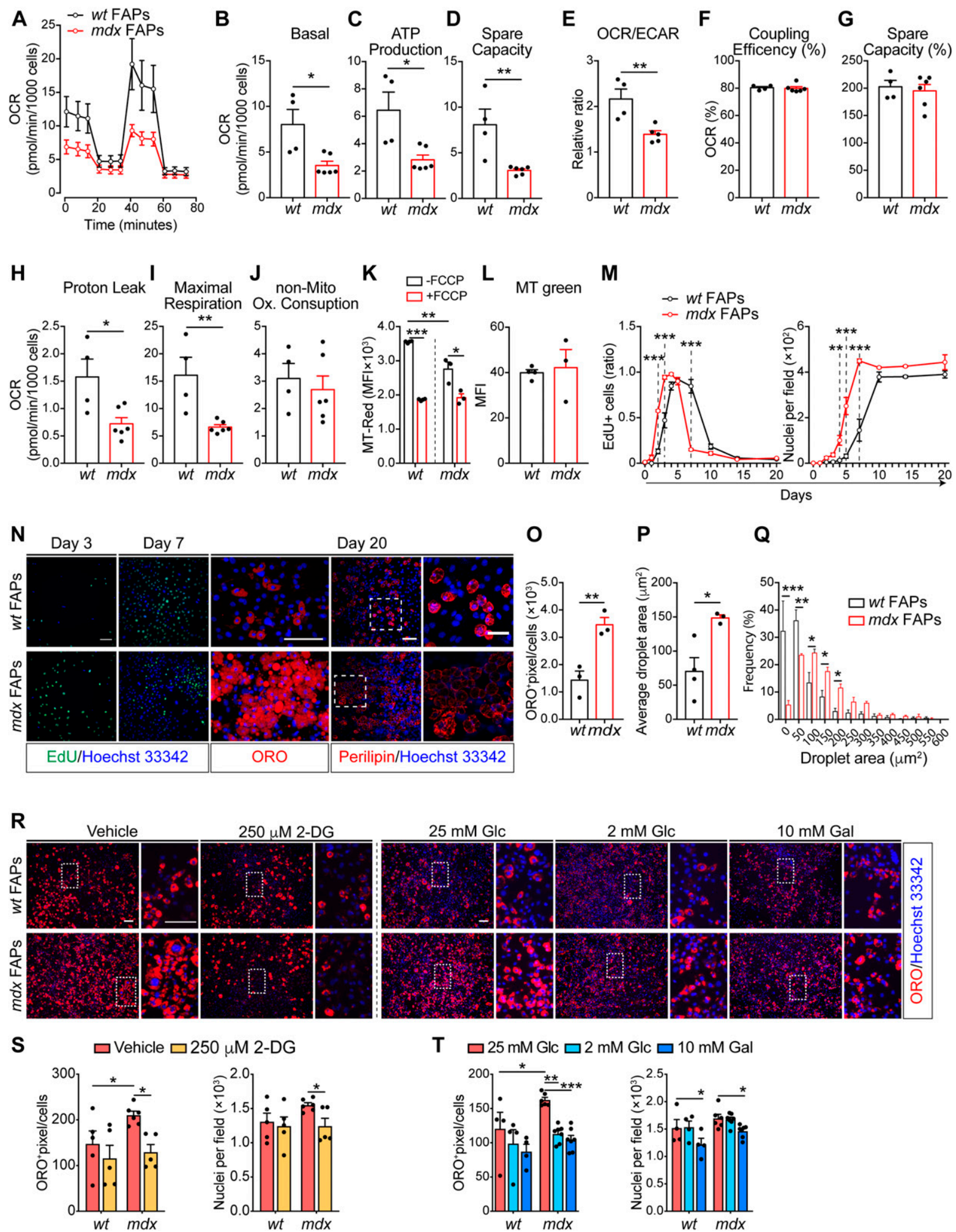

$2 \mathrm{mM} \mathrm{Glc}$

$10 \mathrm{mM} \mathrm{Gal}$

S

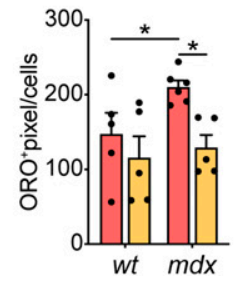

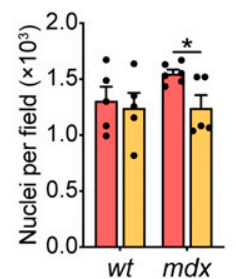
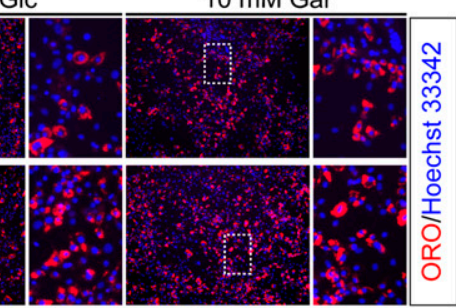

Figure 2. Dystrophic fibro/adipogenic progenitor (FAP) mitochondrial dysfunction correlates with an impaired ex vivo proliferation and adipogenic differentiation that can be modulated by metabolic interventions.

(A) Mitochondrial stress test profile of wt and $m d x$ FAPs (from $45-d$ old wt and $\mathrm{mdx}$ mice) by Seahorse analysis. The oxygen consumption rate (OCR) ( $\mathrm{pmol} / \mathrm{min} / 10^{3} \mathrm{cells}$ ) was monitored for 80 min under basal conditions and upon sequential treatment with the mitochondrial inhibitors oligomycin, FCCP, and rotenone/antimycin (wt FAPs $n=$ 4; $m d x$ FAPs $n=6$ ). (B, C, D, E, F, G, H, I, J) Bar graphs representing basal OCR (B), ATP production (C), spare capacity (D), OCR/extracellular acidification rate ratio (E), coupling efficiency (\% to the basal OCR) (F), spare capacity (\% to the basal OCR) (G), proton leak (H), maximal respiration (I), and non-mitochondrial oxygen consumption 
are less efficient in mitochondrial respiration and favor a glycolytic metabolism.

\section{In vitro metabolic reprogramming of dystrophic FAPs and MuSCs impact on their differentiation potential}

Given the crucial role of the metabolism in controlling the stem cell fate (Knobloch et al, 2017; Pala et al, 2018), we wondered if the altered metabolic state of dystrophic muscle stem cells could impact their ability to proliferate and/or differentiate. In vitrocultured $m d x$ FAPs show an enhanced mitotic rate compared with wt, as revealed by the 5-ethynyl-2'-deoxyuridine (EdU) incorporation and by the growth profile (Fig 2M and N), and reduced doubling time (Fig S3A). In addition, mdx FAPs have a higher adipogenic differentiation potential, as revealed by the increased number of mature adipocytes (Fig 2N and O) with larger lipid droplets, $20 \mathrm{~d}$ after plating (Fig 2N, $\mathrm{P}$, and Q).

To enquire whether the enhanced glycolytic flux in mdx FAPs is responsible for the increase in adipogenic propensity, we monitored wt and mdx FAP adipogenesis in conditions that restrain glycolysis. Specifically, we treated FAPs with $250 \mu \mathrm{M}$ 2-deoxyglucose (2-DG), $2 \mathrm{mM}$ glucose, or $10 \mathrm{mM}$ galactose to inhibit glycolysis and shunt substrates toward OxPhos (Fig 2R). In these experimental conditions, the mitochondria functionality is improved, as shown by the enhanced ATP production (Fig S3B). Remarkably, 2-DG treatment, as well as glucose deprivation, significantly reduce the adipogenic differentiation (Fig 2R-T) and proliferation of $m d x$ FAPS (Fig S3C and D), without affecting their survival (Fig S3E).

We next asked whether the in vitro metabolic reprogramming of wt and dystrophic MuSCs could also impact on their myogenic potential. Whereas culturing MuSCs in the presence of 2-DG does not affect myogenic differentiation (Fig S2K and L), glucose deprivation and galactose treatment significantly increase their ability to form elongated myosin heavy chain (MyHC)-positive myotubes (Fig S2K and M). Although the treatment did not impact on cell viability (Fig S2N-P), MuSC proliferation increases upon glucose reduction and galactose treatment (Fig $\mathrm{S} 2 \mathrm{Q}-\mathrm{S}$ ) as reported by others showing that OxPhos directly influences the differentiation and proliferative capacities of MuSCs (Cerletti et al, 2012).

Overall, our data point to glycolysis as a process which plays a pivotal role in the regulation of dystrophic FAP proliferation and adipogenic differentiation. By contrast, MuSC myogenic differentiation is supported by OxPhos.

\section{A short-term HFD remodels FAP metabolism in $m d x$ mice}

In vitro, metabolic reprogramming of FAPs and MuSCs from $m d x$ mice affects their differentiation potential. Given the crucial role of these progenitor cells in mediating muscle regeneration (Murphy et al, 2011), we next asked whether dietary regimens designed to activate fatty acid oxidation (FAO) and mitochondrial respiration would stimulate and restore OxPhos activity along with the proregenerative potential of $m d x$ FAPs and MuSCs. To this aim, we fed weaned (21-d old) $m d x$ and wt mice for $28 d$ with a standard HFD containing $58 \% \mathrm{kcal}$ in fat. The control group was fed with an isocaloric control/low-fat diet (LFD, $11 \% \mathrm{kcal}$ in fat) (see the Materials and Methods section for details).

During the diet regimen, weight, food and water intake were recorded every $2 \mathrm{~d}$ (Fig S4A). Short-term treatment with the HFD was not sufficient to cause any significant changes in the body, organs, and muscle weight in either group (Fig S4B and C). Nevertheless, after $4 \mathrm{wk}$, cholesterol and triglycerides were significantly increased in both $m d x$ and wt mice on the HFD (Fig S4D).

To elucidate the impact of short-term HFD on FAP and MuSC metabolism, we first profiled the proteome of $w t$ and $m d x$ FAPs and MuSCs under different diet regimens. By applying a label-free liquid chromatography (LC)-MS/MS quantitation approach (Fig 3A) (Kulak et al, 2014; Kelstrup et al, 2018), we were able to quantitate $\sim 4,500$ proteins (Fig S5A). Proteome measurements were highly accurate and reproducible with a Pearson correlation coefficient among biological replicates ranging between 0.85 and 0.95 (Fig S5B). Unsupervised hierarchical clustering (Fig S5B) and principal component analysis (Fig 3B and C) of about 4,500 proteins revealed that the proteome profiles efficiently discriminate different samples according to cell type, genetic background, and diet regimen. Interestingly, the drivers of the discrimination between wt and $m d x$ FAPs (component 1 of the PCA loadings) were significantly enriched for proteins annotated to the cell cycle process, which is known to be up-regulated in dystrophic FAPs (Fig S5C). In addition, we also found that the drivers of the discrimination between HFD and LFD FAPs (component 2 of the PCA loadings) were significantly enriched for oxidative phosphorylation, which we expect to be up-regulated

(J) obtained by Seahorse Wave Desktop software. Statistical significance was estimated by the $t$ test. (K) Bar graph representing the median fluorescence intensity (MFI) of MitoTracker RED (MT-Red) dye in flow cytometry in basal condition and under uncoupling with $10 \mu \mathrm{M} \mathrm{FCCP,} \mathrm{in} \mathrm{wt} \mathrm{and} \mathrm{mdx} \mathrm{FAPs} \mathrm{(} n=3$ ). Statistical significance was estimated by the $t$ test. (L) Bar graph representing median fluorescence intensity (MFI) of MitoTracker GREEN (MT Green) dye in flow cytometry in basal condition on wt and $m d x \operatorname{FAPs}(n=3)$. Statistical significance was estimated by the $t$ test. (M) EdU labelling and growth curve profile of FAPs purified from 45 - $d$ old wt and $m d x$ mice. FAPs were cultured for $20 \mathrm{~d}$ ( $w t n=3 ; m d x n=3$ ). Statistical significance was estimated by two-way ANOVA. (N) Representative EdU (green, $20 \times$ magnification; scale bar, $100 \mu$ m), Oil Red $\mathrm{O}$ (ORO) staining (red, 40× magnification; scale bar, $100 \mu \mathrm{m}$ ), and confocal micrographs of perilipin immunostaining (red, $20 \times$ magnification; scale bar, $70 \mu \mathrm{m}$ ) of FAP cells at 3, 7, and $20 \mathrm{~d}$. Nuclei (blue) were revealed with Hoechst 33342. (N, 0) Bar graph resenting the adipogenic differentiation index of wt and mdx FAPs calculated as OROpositive pixels/cell from the panel $(\mathrm{N})(n=3)$. (N, P) Bar graph representing the average lipid droplet area $\left(\mu \mathrm{m}^{2}\right)$ of confocal images in panel $(\mathrm{N})($ wt $n=4 ; m d x n=3)$. (N, Q) Bar graph representing the frequency distribution of lipid droplet areas of confocal images in panel ( $N)(w t n=4 ; m d x n=3)$. Statistical significance was estimated by $t$ test. (R) Representative ORO staining (10x magnification; scale bar, $100 \mu \mathrm{m}$ ) of FAPs from 45-d-old wt and mdx mice. Adipogenic differentiation was obtained by incubating FAPS in adipocyte differentiation medium (ADM) followed by the adipocyte maintenance medium (AMM) in the presence of 25 mM glucose (GIC) supplemented with DMSO (vehicle) or $250 \mu \mathrm{M}$ 2-deoxyglucose (2-DG). Alternatively, FAPs were differentiated by incubating cells with the opportune differentiation media containing either 25 mM Glc, 2 mM Glc, or 10 mM galactose (Gal). Insets are enlarged views of the dashed areas (scale bar: $100 \mu \mathrm{m}$ ). Nuclei (blue) were revealed with Hoechst 33342 . (S) Bar plots reporting the adipogenic index (left) and the average number of nuclei per field (right) for FAPs differentiated in the presence of the vehicle or $250 \mu \mathrm{M} 2$-DG treatment. (T) Bar plots reporting the adipogenic index (left) and the average number of nuclei per field (right) for FAPs differentiated in the presence of $25 \mathrm{mM}$ Glc, $2 \mathrm{mM}$ Glc, or $10 \mathrm{mM}$ Gal treatment (wt $n=3 ; m d x n=3$ ). Statistical significance was estimated by two-way ANOVA. All data are represented as mean \pm SEM and statistical significance is defined as ${ }^{*} P<0.05 ;{ }^{* *} P<0.01 ;{ }^{* *} P<0.001$. 
A

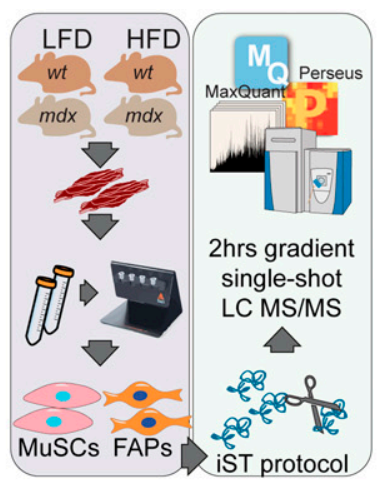

B

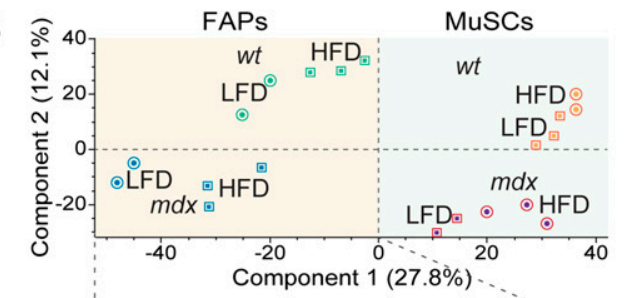

C

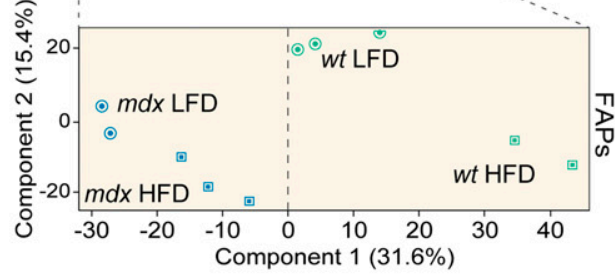

E

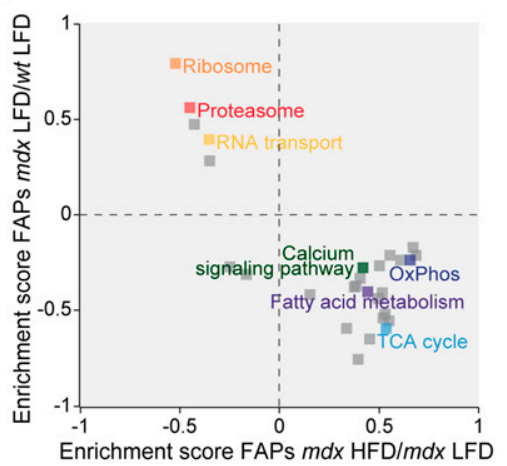

Figure 3. Short-term high-fat diet (HFD) rewires the metabolic signature of dystrophic fibro/adipogenic progenitors (FAPs).

(A) Experimental workflow to analyze the proteome of FAPs and Muscle Satellite Cells purified from 49- $d-$ old wt and $m d x$ mice fed with low-fat diet (LFD) and HFD (wt LFD $n=3$; wt HFD $n=2 ; m d x$ LFD $n=2 ; m d x \operatorname{HFD}$ $n=3)$. (B) Principal component analysis of the proteomic profiles of FAPs and muscle satellite cells from mice under LFD or HFD. (C) The principal component analysis inset shows the sample separation of wt/mdx FAPs from mice fed with LFD and HFD. (D) Scatterplot of the log2 fold change of protein expression level of 480 proteins significantly modulated in $m d x /$ wt FAPs ( $y$-axis) and HFD/LFD $m d x$ FAPs (x-axis). (E) Two-dimensional annotation enrichment analysis of the significantly modulated proteins in $m d x /$ wt FAPs ( $y$-axis) and HFD/LFD $m d x$ FAPs ( $\mathrm{x}$-axis). Groups of related GO terms are labelled with the same color, as described in the inset. (F) Quantitation of the ATP in FAPs purified from 49-d-old $m d x$ mice fed with LFD and HFD $(n=3)$. (G) Mitochondrial stress test profile of LFD and HFD $m d x$ FAPs. Oxygen consumption rate ( $\mathrm{pmol} / \mathrm{min} / 10^{3}$ cells) was monitored in real time (for $80 \mathrm{~min}$ ) under basal condition and upon sequential treatment with mitochondrial inhibitors ( $m d x \operatorname{LFD} n=4 ; m d x \operatorname{HFD} n=4)$. (H, I) Bar graphs representing basal oxygen consumption rate $(\mathrm{H})$ and ATP production (I). (J, K) Western blot (J) and relative densitometric analysis (K) of PKM2 and vinculin in FAPs from $m d x$ mice fed with LFD and HFD ( $m d x$ LFD $n=4$; $m d x$ HFD $n=4$ ). Statistical significance was estimated by $t$ test. All data are represented as mean $\pm \mathrm{SEM}$ and statistical significance is defined as ${ }^{*} P<0.05$; ${ }^{* *} P<0.01$; ${ }^{* * *} P<0.001$.
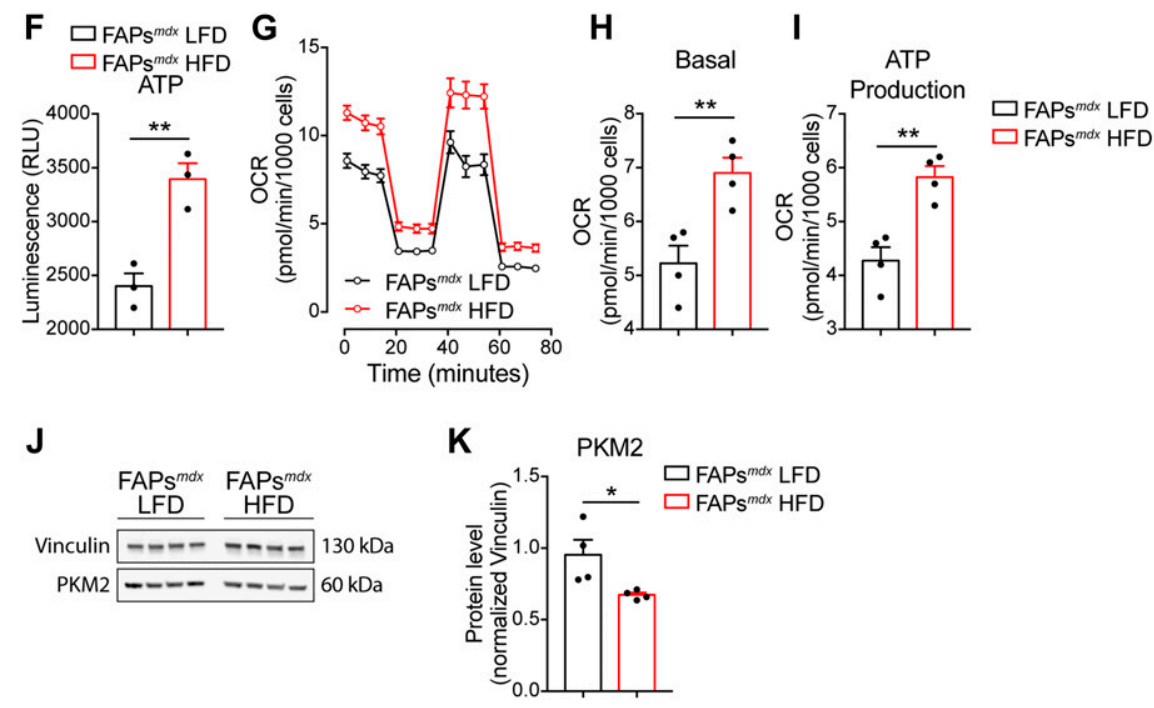

in response to the HFD treatment (Fig S5C). About 9\% of the proteome of both dystrophic FAPs and MuSCs was found to be significantly modulated by the diet (Fig S5D and E). The HFD significantly increased the level of proteins involved in fatty acid metabolism and OxPhos in FAPs as well as, albeit to a lesser extent, in MuSCs of dystrophic mice (Fig S5F).

We next focused on those proteins that were significantly modulated in the $m d x$ model when compared with wt. Interestingly, in both FAPs (Fig 3D) and MuSCs (Fig S5G), we observed an inverse correlation ( $P C=-0.6$ in FAPS, $P C=-0.7$ in MuSCS) between the dystrophy-dependent proteome changes ( $m d x$ versus $w t)$ and the diet-dependent proteome modulation ( $m d x$ HFD versus $m d x$ LFD). This observation suggests that the HFD restores in $m d x$ progenitor cells a proteome profile that is similar to the wt counterpart. We next used the two-dimensional annotation enrichment analyses to investigate, which biological processes were mainly restored by 
HFD in mdx FAPs and MuSCs. Proteins involved in proteinelongation and translation, here annotated as "ribosomal," were up-regulated in $\mathrm{mdx}$ cells and reduced in concentration upon HFD (Figs $3 \mathrm{E}$ and $\mathrm{S} 5 \mathrm{H}$ ). In agreement with our previous observations, HFD restores the expression levels of mitochondrial proteins in both dystrophic FAPs and MuSCs, whereas FAO and OxPhos were significantly enriched only in $m d x$ FAPs (Figs $3 E$ and S5H). In agreement, most of the enzymes involved in the TCA cycle, OxPhos, and FAO were significantly up-regulated (log2 of the median fold change $=0.8$ ) by the HFD treatment only in dystrophic FAPs and not in MuSCs (Fig S5I and J). In line with these findings, we also observed that HFD treatment significantly up-regulated PDK4, by 16-fold, in dystrophic FAPs and not in MuSCs (Fig S5K). PDK4 is a key metabolic enzyme enhancing FAO utilization through its inhibitory activity on pyruvate dehydrogenase (Palamiuc et al, 2015).

Prompted by the observed modulation of key metabolic enzymes, we asked whether the changes in the proteome profile, induced by the short-term HFD, cause a reprogramming of the metabolism in dystrophic FAPs and overcome their mitochondrial defect. To address this point, we measured the ATP levels and characterized the mitochondrial bioenergetics in cultured FAPS purified from muscles of dystrophic mice fed with HFD and LFD. Remarkably, HFD treatment increased the ATP production and improved the mitochondrial functionality of FAPS (Fig 3F-I). Consistently, the levels of PKM2 were blunted (Fig 3J and K), indicating that HFD favors the oxidative processes at the expense of glycolysis.

\section{Short-term HFD restores key regulatory signaling networks in $m d x$ FAPs}

We next asked whether, in addition to metabolism, the HFD would also affect key signaling pathways in mdx mice. To this end, we first selected the proteins whose abundance was affected in $m d x$ and restored to values closer to wt by the diet. 220 and 283 proteins have such characteristics in MuSCs and FAPs, respectively (Fig S6A). Next, we mapped this subset of proteins onto a literature-derived network (Sacco et al, 2016) of signaling and physical interactions extracted from the SIGNOR (Perfetto et al, 2015) and the Mentha databases (Calderone et al, 2013). This strategy revealed key signaling networks and protein complexes whose concentrations were affected by the HFD only in dystrophic FAPs and not wt FAPs and in MuSCs (Figs S6B, S7A and B, and S8A and B). This network analysis showed that HFD treatment in dystrophic cells restores to wt level the abundance of the sarcolemmal protein, dysferlin, which plays an important role in the control of $\mathrm{Ca}^{2+}$-dependent sarcolemmal stability and resealing (Han et al, 2011). Our network analysis enabled us to uncover that the HFD treatment decreases the concentration levels of key proteins positively controlling FAP proliferation, which is pathologically enhanced in dystrophic muscle (Fig 4A) (Lemos et al, 2015; Marinkovic et al, 2019). Consistently, we observed a significant reduction in the number of PDGFR $\alpha$-positive FAPs by labelling tibialis anterior (TA) muscle sections from dystrophic mice fed with HFD (Fig 4B). As inferred from the network modelling approach, HFD significantly suppresses the fraction of FAPs expressing the proliferation marker Ki67 (Fig 4B-D).

Interestingly, we also observed that the HFD treatment causes an up-regulation of $\beta$-catenin (Figs 4A, E, and F, and S7B), a crucial hub controlling a variety of biological processes, including the expression level of follistatin (Jones et al, 2015) known to mediate some of the promyogenic effects of FAPs (Mozzetta et al, 2013). Consistently, two negative regulators of $\beta$-catenin, MEST/PEG1 ( $\mathrm{Li}$ et al, 2014) and casein kinase $1 \alpha$ (Amit et al, 2002), are expressed at higher levels in mdx FAPs, whereas their modulation is reverted in FAPs from mice fed with HFD (Fig 4A, E, and F).

Next, we investigated whether the increased HFD-dependent $\beta$-catenin expression was also associated to an up-regulation of follistatin, which is significantly decreased in dystrophic FAPs in comparison with wt (Fig 4G). In agreement with our hypothesis, the HFD restored the mRNA of follistatin to wild-type levels (Fig 4G). In addition, we demonstrated that in vitro treatment of dystrophic FAPs with a mixture of fatty acids ( $50 \mu \mathrm{M}$ of BSA-coupled palmitate, $50 \mu \mathrm{M}$ of BSA-coupled oleate, and $100 \mu \mathrm{M}$ carnitine) (Fig $4 \mathrm{H}$ ) increase Ctnnb1 and Fst gene expression (Fig 4I).

To demonstrate the positive relation between $\beta$-catenin and follistatin in FAPS, we stabilized $\beta$-catenin through the highselective GSK3 inhibitor, LY2090314 (Fig 4J) (Rizzieri et al, 2016; Kunnimalaiyaan et al, 2018). GSK3 blockade stabilizes enhances Ctnnb1 expression at 48 and $72 \mathrm{~h}$ while inducing a peak of Fst after $48 \mathrm{~h}$ of treatments (Fig 4K).

These observations encouraged us to investigate whether the HFD could increase the ability of FAPs to promote myogenic differentiation of MuSCs from dystrophic mice. Conditioned media (CM) of FAPs from mice fed with HFD are enriched in follistatin compared with LFD (Fig 4L). Independently from the diet treatment, CM from FAPs are able to promote the differentiation of MuSCs. However, media conditioned with FAPs from mice fed with the HFD better promote myogenesis of mdx MuSCs, when compared with media conditioned with LFD FAPs (Fig 4M-O).

Altogether, our data support a model whereby the in vivo metabolic reprogramming suppresses the FAP aberrant proliferation in dystrophic mice, while enhancing their ability to promote myogenesis of MuSCs.

\section{A short-term HFD ameliorates the dystrophic phenotype}

Finally, we asked whether the metabolic reprogramming of muscle progenitor cells mediated by the HFD would also have an impact on the histological phenotype of dystrophic muscles. First, we monitored the serum level of creatine phosphokinase, a sensitive marker of muscle damage. Short-term HFD reduced serum creatine phosphokinase, suggesting that HFD protects $m d x$ muscles from dystrophic damage (Fig 5A). Consistently, the incidence of centronuclear myofibers is reduced in dystrophic TAs and diaphragms upon HFD treatment (Fig 5B-E). Given the key role of FAPs in mediating fibrosis (Uezumi et al, 2011; Hogarth et al, 2019) in degenerating muscles, we next asked whether HFD could also ameliorate fibrosis in TAs and diaphragms. HFD decreases the abundance of fibrotic scars both in the TA and diaphragm muscles (Fig 5F-I). Intramuscular FAP-derived adipocytes are also common during the late stages of muscular dystrophy but absent in young mouse muscles (Uezumi et al, 2011; Hogarth et al, 2019). However, rare adipogenic depots are found in diaphragm muscles, independently from mouse genotypes and diet regimen (Fig S9A and B). 
A

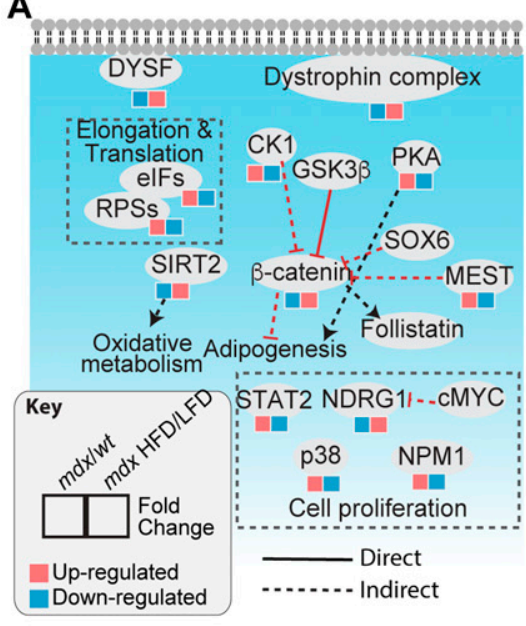

B
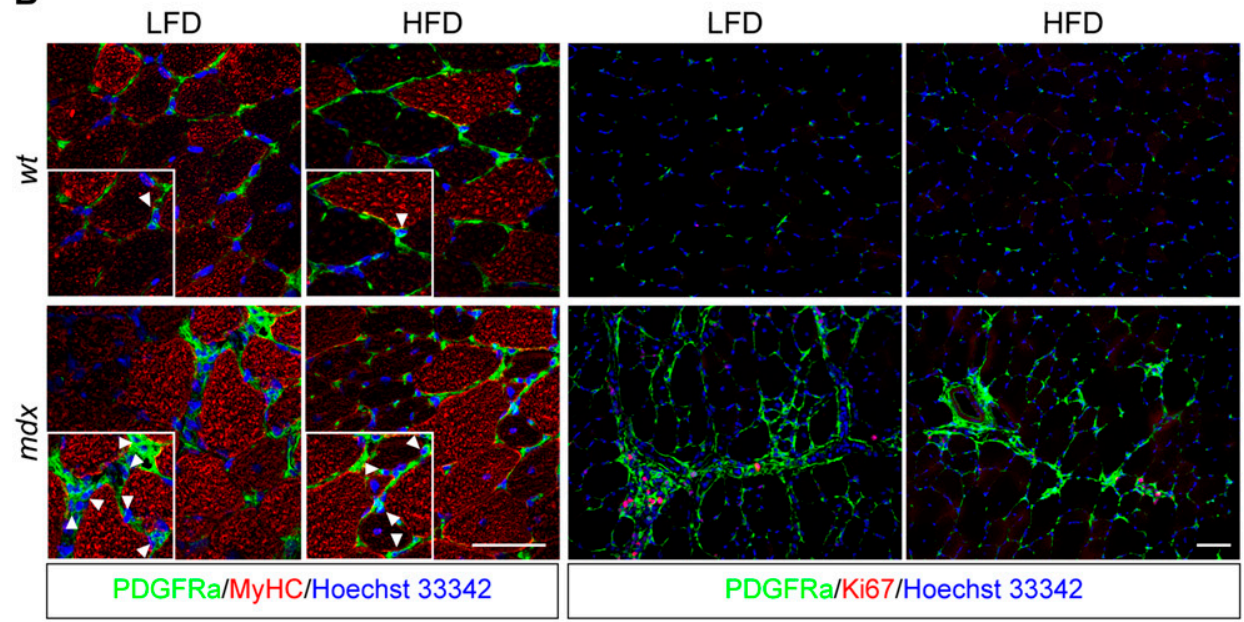

C

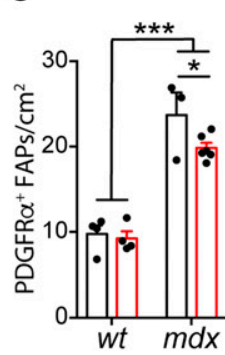

H

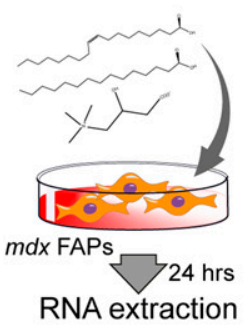

D

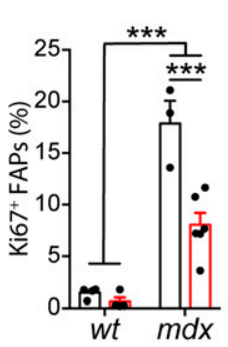

E

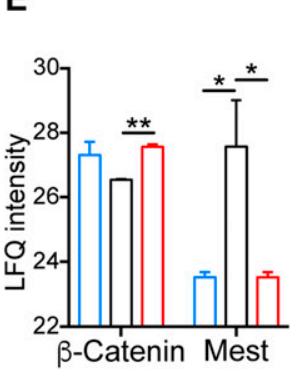

F

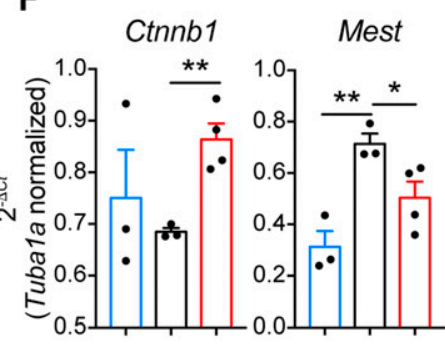

G

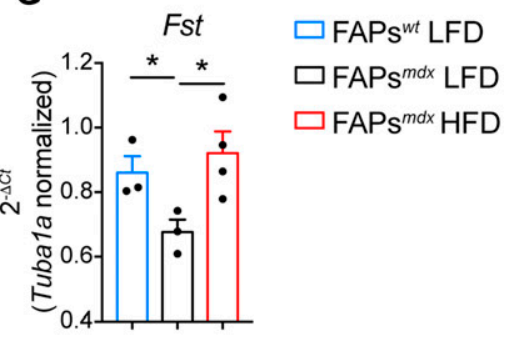

K Ctnnb1 Fst

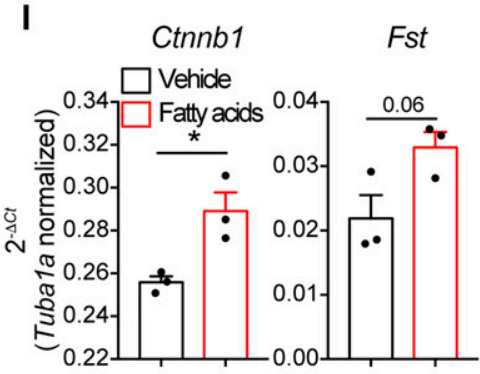

J
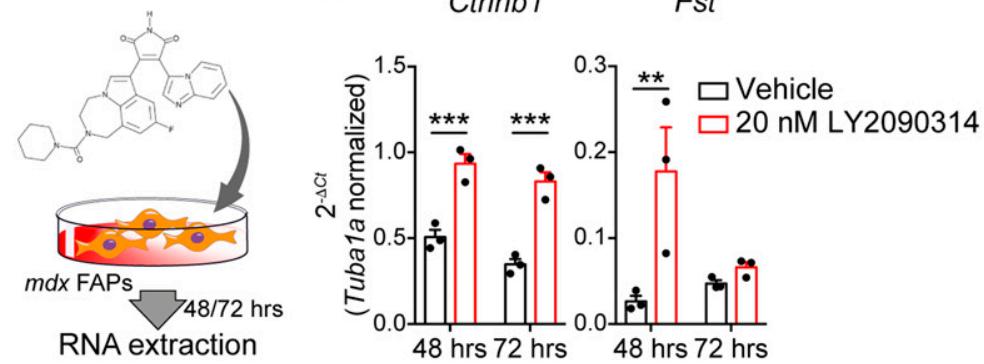

FAP culture supernatants

M

$\mathbf{N}$
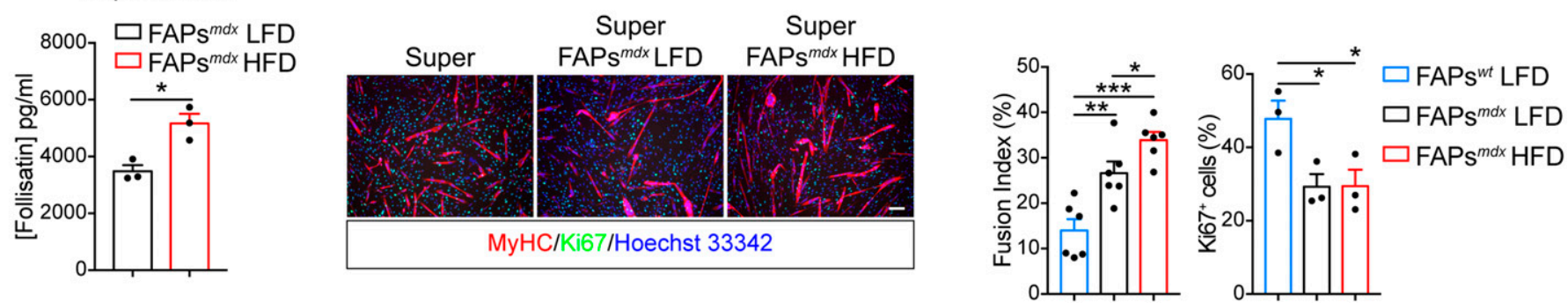

Figure 4. Short-term high-fat diet (HFD) limits fibro/adipogenic progenitor (FAP) persistence in dystrophic muscles and restores $\beta$-catenin expression enhancing their promyogenic abilities.

(A) Schematic representation of the main molecular events reverted by HFD treatment in mdx FAPs. (B) Representative confocal images of PDGFR $\alpha$-positive FAPs (green) from 49-d-old wt and $m d x$ mice fed with low-fat diet (LFD) and HFD (60x magnification; scale bar, $20 \mu \mathrm{m}$ ). Fibers (red) were stained using antibodies directed against the MyHC isoforms. Representative micrograph (20× magnification; scale bar, $100 \mu \mathrm{m}$ ) showing proliferating FAPs by coupling PDGFR staining (green) with anti-Ki67 antibodies (red). Nuclei (blue) were revealed with Hoechst 33342. (C) Bar plot reporting the number of PDGFR $\alpha$-positive FAPs per $\mathrm{cm}^{2}$ of muscle section (wt LFD $n=4$; wt 
To further elucidate the role of HFD on muscle regeneration in dystrophic mice, we examined the cross-sectional area (CSA) of TAs (Fig 5J). This analysis highlighted a rescue in the $m d x$ TA myofiber calibers whose average area approaches wt level (Fig 5K) with a significant reduction of the number of small fibers and a corresponding increase of fibers of larger calibers (Fig S9C). Although evident in TAs, such hypertrophic improvement was not appreciable in diaphragm muscles (Fig S9D-F).

The increased CSA of $m d x$ TA muscles upon HFD did not result from an increased commitment and/or differentiation of MuSCs because, when cultured ex vivo, MyoD expression levels, EdU incorporation, and the fusion index do not differ, comparing MuSCs from LFD- or HFD-fed mice both at early and late culture time (Fig $\mathrm{S} 9 \mathrm{G}$ and $\mathrm{H}$ ). At the molecular level, we found that the increased CSA parallels an activation of the MTOR pathway, as revealed the enhanced phosphorylation of mTOR and its indirect downstream target RPS6, in whole muscle lysates (Fig 5L-N). This observation is consistent with the HFD-dependent increase of the expression level of the hypertrophic cytokine IGF1 in dystrophic FAPs (Fig 50). Overall, we found that short-term HFD is able to ameliorate the dystrophic phenotype while triggering a hypertrophic response via the mTOR pathway.

\section{FAPs mediate the beneficial effects of HFD}

Follistatin and IGF1 are known to mediate hypertrophy in skeletal muscle and their expression is enhanced in FAPs after short-term HFD. To ask whether the hypertrophic phenotype, observed in the $m d x$ HFD muscles, can be mediated by FAPs, we transplanted $m d x$ FAPS from HFD-fed $m d x$ mice into the TA of syngeneic dystrophic mice. To this end, purified mdx FAPs from LFD- and HFD-fed mice were transduced with a lentivirus carrying a GFP-expressing vector, to allow in vivo tracing (Fig 6A). After $15 \mathrm{~d}$, GFP-positive cells were found interspersed in the muscle interstitium (Fig 6B). GFP-positive cells also co-expressed the FAP distinctive marker PDGFR $\alpha$, confirming that transplanted FAPs were successfully engrafted into the host muscles (Fig 6C). Remarkably, the limb receiving FAPs from HFD-fed mice displayed an increased CSA in comparison with the LFD-receiving limb (Fig 6D-G), supporting the conclusion that metabolically reprogrammed FAPs can improve the dystrophic phenotype.

These observations are consistent with a model whereby the HFD-dependent metabolic reprogramming of FAPs is responsible for the amelioration of the dystrophic phenotype that is observed in $m d x$ mice fed on the HFD (Fig 7).

\section{Discussion}

Metabolism plays a crucial role in controlling the fate of progenitor cells in tissue development, homeostasis, regeneration, and disease (Lunt \& Vander Heiden, 2011; Ryall et al, 2015; Knobloch et al, 2017; Joseph et al, 2018; Pala et al, 2018; Marinkovic et al, 2019). In the skeletal muscle, a variety of nutritional approaches, including caloric restriction, fasting-mimicking drugs, long-term (16 wk) HFD, and amino acid treatments have been applied, displaying different effects on healthy and dystrophic muscle regeneration (RadleyCrabb et al, 2011; Cerletti et al, 2012; Banfi et al, 2018; Juban et al, 2018).

However, the molecular and cellular mechanisms linking metabolic reprogramming to its impact on muscle regeneration are still poorly characterized. In this context, it has been reported that the metabolic reprogramming of MuSCs tunes up the stem cells for the transition from quiescence to activation through the epigenetic control mediated by NAD-dependent deacetylase SIRT1 (Ryall et al, 2015). Moreover, mitochondrial and peroxisomal FAO are key determinants of MuSCs stem cell fate throughout the embryonal and postnatal development (Pala et al, 2018).

Here, we applied systematic approaches to investigate the role of metabolism in controlling the fate of muscle interstitial FAPs and its impact on muscle physiology and regeneration. In healthy individuals, the differentiation multipotency of FAPs is restrained by autocrine and paracrine signals (Joe et al, 2010; Uezumi et al, 2010; Lees-Shepard et al, 2018; Marinkovic et al, 2019; Reggio et al, 2019a). However, when muscle damage set regeneration in motion, FAPs release key paracrine signals targeting MuSCs and other cell partners, to trigger tissue repair (Christov et al, 2007; Joe et al, 2010; Uezumi et al, 2010; Murphy et al, 2011; Heredia et al, 2013; Lemos et al, 2015; Kuswanto et al, 2016). In pathological conditions or aging, the progressive deterioration of the mechanisms keeping FAP differentiation in check, leads to their unrestrained differentiation into fibroblasts or adipocytes, causing the formation of fibrotic scars and/or fat deposition. This outcome irreversibly alters muscle functionality (Uezumi et al, 2011; Kopinke et al, 2017; Marinkovic et al, 2019).

We have recently applied high-resolution MS-based proteomics to profile the proteome changes occurring in dystrophic FAPS isolated from mdx mice (Marinkovic et al, 2019). Here, we show that key metabolic enzymes involved in glycolysis are up-regulated in dystrophic FAPs, whereas the abundance of TCA and OxPhos enzymes is reduced in comparison with wild-type cells. We have further investigated the metabolic state of wild-type and dystrophic

HFD $n=4 ; m d x$ LFD $n=3 ; m d x$ HFD $n=6$ ). (D) Bar plot reporting the fraction of Ki67-positive cells in PDGFR $\alpha$-positive FAPs in TA cross-sections ( $w t$ LFD $n=4 ;$ wt HFD $n=4$; $m d x \operatorname{LFD} n=3 ; m d x$ HFD $n=6$ ). Statistical significance was estimated by two-way ANOVA. (E) Mass spectrometry-based quantitation of $\beta$-catenin and Mest in wt and $m d x$ FAPs from mice fed with LFD and HFD. (F) Quantitative PCR for $\beta$-catenin and Mest in mdx FAPs from mice fed with HFD and LFD mice. (G) Quantitative PCR of Follistatin in wt and $m d x$ FAPs from mice fed with LFD and HFD (wt LFD $n=3$ mice; $m d x$ LFD $n=3$ mice; $m d x$ HFD $n=4$ mice). Statistical significance was estimated by one-way ANOVA. (H) Representative scheme summarizing the experimental procedure to treat, ex vivo, FAPs with BSA-coupled palmitate/oleate ( $50 \mu \mathrm{M} / 50 \mu \mathrm{M})$ and $100 \mu \mathrm{M}$ carnitine. ( $\mathbf{H}, \mathbf{I})$ Quantitative PCR of Ctnnb1 and Fst transcripts in mdx FAPs treated as shown in (H). Statistical significance was estimated by $t$ test. (J) Representative scheme summarizing the experimental procedure to treat, ex vivo, mdx FAPs with 20 nM LY2090314. (K) Quantitative PCR of Ctnnb1 and Fst transcripts in mdx FAPs treated with 20 nM LY2090314 for 48 and $72 \mathrm{~h}$. Statistical significance was estimated by Two-way ANOVA $(n=3)$. (L) Bar plot reporting the concentrations of Follistatin in FAP-derived supernatants. Follistatin concentrations were analyzed via ELISA assay. (M) Representative immunofluorescence (20× magnification; scale bar, $100 \mu$ m) of muscle satellite cell (MuSC)-derived myotubes (red) upon incubation with the control and LFD/HFD mdx FAP-derived supernatants. Proliferating myoblasts (green) were detected using a Ki67 specific antibody. (N) Bar plot reporting the fusion index $(n=6)$ of differentiated MuSCs in each treatment condition. (0) Bar plot reporting the fraction of Ki67positive MuSCs in each treatment condition. Statistical significance was estimated by One-way ANOVA. All data are represented as mean \pm SEM and Statistical significance is defined as ${ }^{*} P<0.05 ;{ }^{* *} P<0.01 ;{ }^{* * *} P<0.001$ 
A

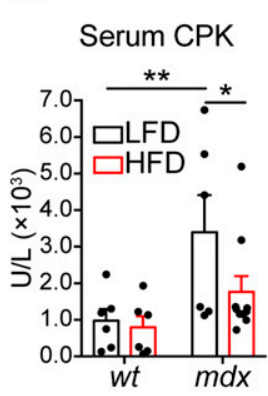

B

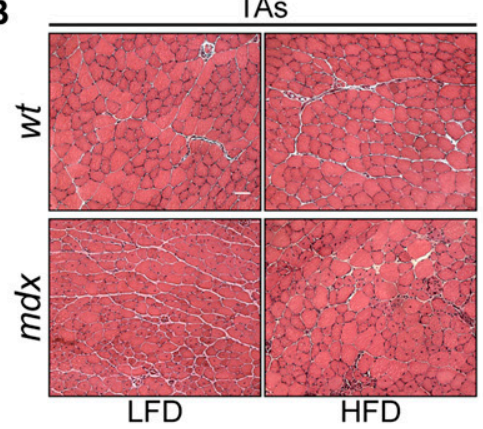

C

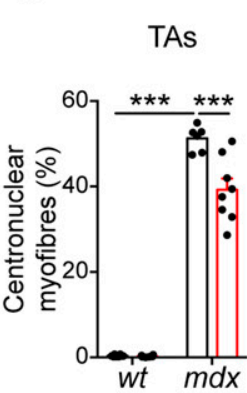

D

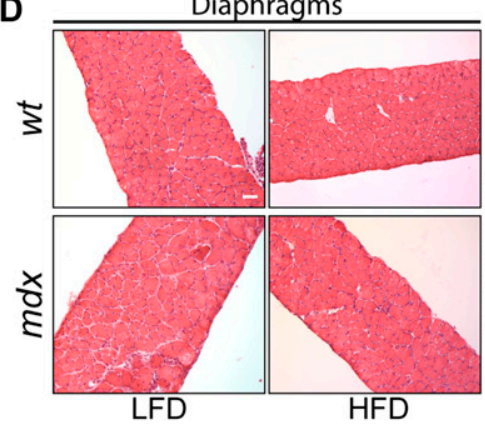

E

Diaphragms

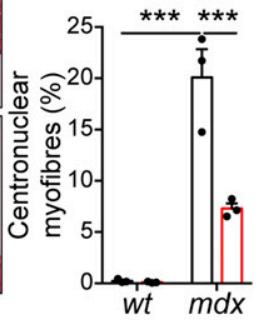

F

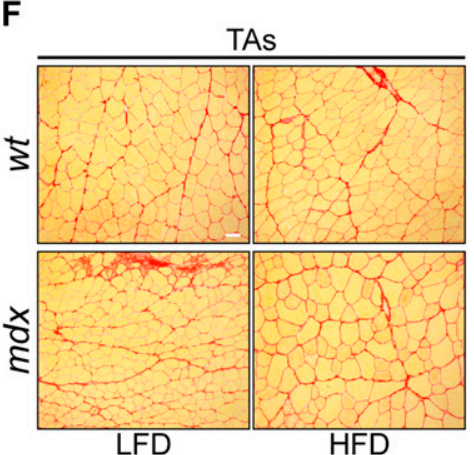

G

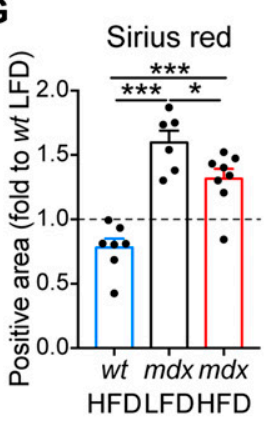

H

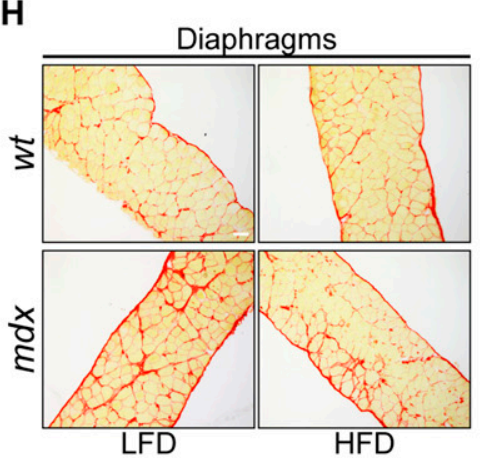

I

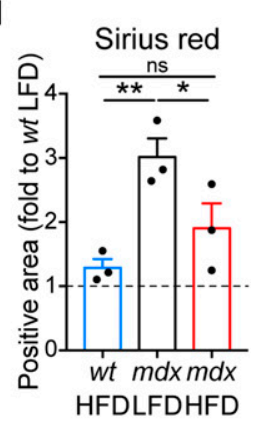

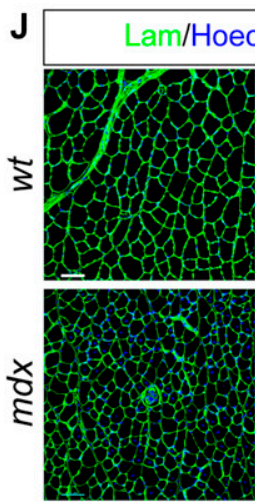

LFD

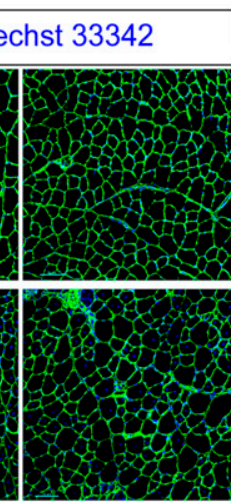

HFD

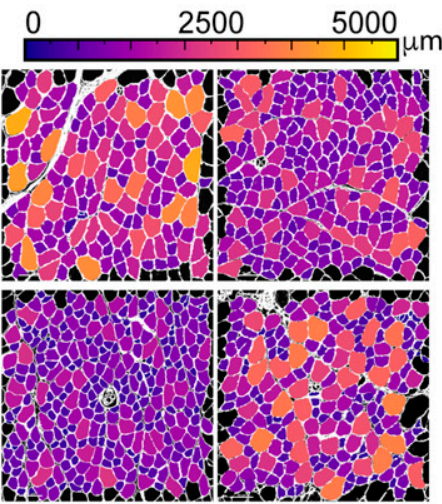

LFD

HFD
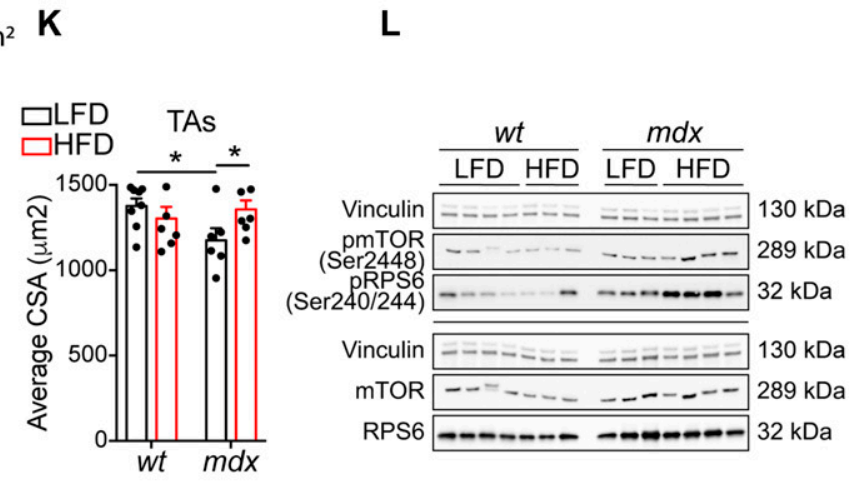

M

phospho-mTOR $\mathbf{N}$ phospho-RPS6
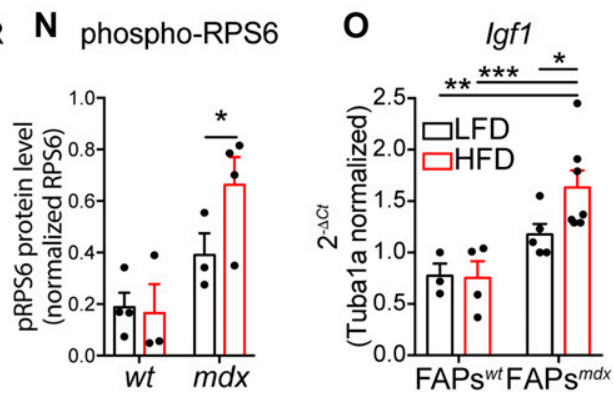

L

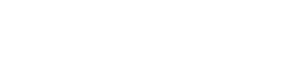
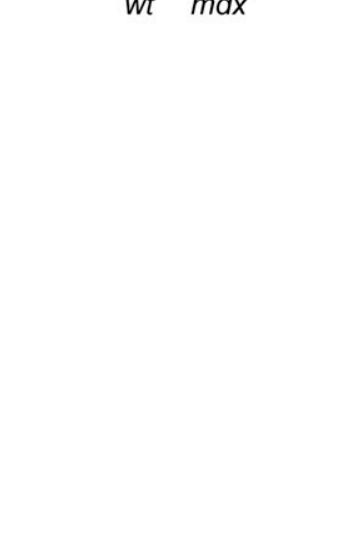

Figure 5. Short-term high-fat diet (HFD) ameliorates the $m d x$ phenotype.

(A) Serum creatine phosphokinase (units per liter, U/l) from 49-d-old wt and $m d x$ mice fed with low-fat diet (LFD) or HFD ( $w t$ LFD $n=6$; wt HFD $n=7$; $m d x$ LFD $n=6$; $m d x$ HFD $n=10$ ). (B) Representative hematoxylin and eosin staining of TA cross-sections from 49-d-old wt and mdx mice fed with LFD or HFD (20× magnification; scale bar, 100 $\mu m$ ). (C) Percentage of centrally nucleated myofibers in TAs ( $w t$ LFD $n=8 ; w t$ HFD $n=6 ; m d x \operatorname{LFD} n=6 ; m d x$ HFD $n=8$ ). (D) Representative hematoxylin and eosin staining of diaphragm cross-sections from 49-d-old wt and $m d x$ mice fed with LFD or HFD (20x magnification; scale bar, $100 \mu \mathrm{m}$ ). (E) Percentage of centrally nucleated myofibers in diaphragms ( $w t$ LFD $n=3 ;$ wt HFD $n=3 ; m d x \operatorname{LFD} n=3 ; m d x \operatorname{HFD} n=3$ ). Statistical significance was estimated by Two-way ANOVA. (F) Representative picrosirius red staining of TA cross-sections (20x magnification; scale bar, 100 im). (G) Bar plot showing the extent of picrosirius red area in TA cross-sections from 49-d-old wt and $m d x$ mice fed 
FAPs. Our analysis reveals that wild-type FAPs, similarly to other stem progenitors, mainly rely on FAO to maintain their quiescence (Ryall et al, 2015; Knobloch et al, 2017). By contrast, dystrophic FAPs, alike dystrophic fibers, have a reduced mitochondrial functionality and mainly rely on glycolysis to generate ATP (Onopiuk et al, 2009; Timpani et al, 2015).

Increasing evidence implicates modulation of glycolysis and OxPhos fluxes in the regulation of stem cell proliferation and differentiation (Ryall et al, 2015; Knobloch et al, 2017; Pala et al, 2018). Our in vitro and in vivo data support a crucial role for metabolism in the regulation of FAP biology. Modulating FAP metabolism by limiting glycolysis or enhancing mitochondrial OxPhos not only impacts cell proliferation but also corrects the aberrant activation and adipogenic differentiation of dystrophic FAPS.

Similarly to FAPS, dystrophic MuSCs show mitochondrial defects and mainly rely on glycolysis for energy production. This observation is consistent with a report showing that myoblasts derived from $m d x$ mice have a reduced oxygen consumption and increased glycolysis (Onopiuk et al, 2009; Timpani et al, 2015). Interestingly, our functional and biochemical assays suggest that the metabolic differences observed between dystrophic and wild-type FAPs are more pronounced than those observed in MuSCs. In agreement with this observation, an in vivo short-term HFD treatment of dystrophic mice significantly modulates the abundance of metabolic enzymes in FAPs, although only slightly affecting MuSCs. The dramatic differences in the proteome profile of FAPs and MuSCS (more than $42 \%$ of the proteins are significantly modulated) may explain the different sensitivity of these two cell types to the diet.

Our analysis not only demonstrates an important effect of the diet on FAP metabolism but also reveals that a short-term HFD causes a significant rewire of key signaling networks.

Namely, we found that dystrophic FAPs have a reduction in the abundance of DAPC and dysferlin, similar to what has been already shown in mdx muscle fibers (Ohlendieck \& Campbell, 1991). Interestingly, we observed that the HFD regimen restores the expression level of DAPC and dysferlin in FAPS, suggesting a correcting effect on intracellular calcium homeostasis by the activation of the surface membrane repair (Bansal et al, 2003). Because dystrophin deficiency has been already associated with myofiber mitochondrial dysfunctions (Timpani et al, 2015), the HFD-dependent recovery of the dystrophin complex in FAPs could represent one of the mechanisms contributing to the improved mitochondrial functionality. The reduced glycolytic activity of dystrophic FAPs upon HFD treatment is associated with a change in the abundance of important cell cycle regulators, including the decreased expression of p38, NPM1, STAT2, and the increase of the sirtuin SIRT2 and NDRG1, a tumor suppressor negatively regulated by CMYC (Zhang et al, 2008). Indeed, we observed a significant reduction of PDGFR $\alpha$-positive FAPs in the muscle interstitium of dystrophic mice fed with HFD as compared with the LFD counterpart. This observation suggests that HFD may attenuate FAP hyper-proliferation in dystrophic muscles.

Importantly, our network analysis revealed that the crucial hub $\beta$-catenin is also positively modulated by the diet in dystrophic FAPs. The transcriptional coactivator $\beta$-catenin plays a key role in controlling several biological processes in mesenchymal cells, including adipogenesis and fibrogenesis (Rudolf et al, 2016; Judson et al, 2018). Interestingly, HFD was already shown to regulate the expression of members of the Wnt signaling pathway in rodent models of diabetes and obesity (Chen et al, 2015). In agreement with this observation, an HFD suppresses the activity of GSK3 $\beta$ in an AKT-dependent manner (Wang et al, 2015). Interestingly, Anderson et al (2008) showed that GSK3 $\beta$ could also control mitochondrial functionality through its negative activity on PGC1a (Anderson et al, 2008). However, it is not clear yet whether the HFD treatment controls $\beta$-catenin activation via cell autonomous or non-cell autonomous mechanisms.

Noteworthy, $\beta$-catenin has been involved in the regulation of the expression of follistatin, a key myokine with promyogenic activity in MuSCs (Jones et al, 2015). Here, we demonstrate that the HFDdependent modulation of $\beta$-catenin restores follistatin expression, which is compromised in dystrophic FAPs. Similarly, we observed that IGF1 expression was also restored by HFD in dystrophic FAPS.

IGF1 and follistatin play a key role in promoting myogenesis (Latres et al, 2005) and could stimulate myogenesis under an HFD regimen. Here, we observed the activation of the mTOR-dependent pathway that, in response to increased IGF1 production, stimulates myofiber hypertrophy. By contrast, in MuSCs isolated and differentiated ex vivo, not exposed to FAP-derived signals, the hypertrophic response is not observed. In support of this model, the supernatant of FAPs from HFD-fed $m d x$ mice promotes MuSC-myotube formation more efficiently than media from control/LFD $m d x$ cells. The HFDdependent increase in MuSC myogenic activity parallels a significant amelioration of the dystrophic muscle phenotype, as highlighted by the reduced incidence of centrally nucleated myofibers, and the increase in fiber CSA. The causal link between the metabolic reprogramming and the dystrophic phenotype amelioration can be recapitulated only by injecting the HFD-derived FAPs. This observation demonstrates that FAP metabolic plasticity plays a crucial role in mediating the beneficial effects of HFD.

In agreement with this evidence, our proteomic data combined with functional metabolic analysis support a model whereby a short-term HFD reprograms the metabolism of FAPS. Upon HFD treatment, dystrophic FAPs change their metabolic state and regain their positive regulatory role in promoting muscle regeneration, by

with LFD or HFD (wt LFD $n=8$; wt HFD $n=7 ; m d x$ LFD $n=6 ; m d x$ HFD $n=8$ ). (H) Representative picrosirius red staining of diaphragm cross-sections from 49-d-old wt and mdx mice fed with LFD or HFD (20x magnification; scale bar, $100 \mu \mathrm{m})$. (I) Bar plot showing the extent of picrosirius red area in diaphragm cross-sections (wt LFD $n=3$; wt HFD $n=3 ; m d x$ LFD $n=3 ; m d x$ HFD $n=3$ ). Statistical significance was estimated by one-way ANOVA. (J) Representative confocal micrographs of laminin-stained (green) TA crosssections from 49-d-old wt and $m d x$ mice fed with LFD or HFD (20x magnification; scale bar, $70 \mu$ m), left panel. Pseudo-color representation of the myofiber caliber, ranging from 0 to $5,000 \mu \mathrm{m}^{2}$, right panel. Nuclei (blue) were revealed with Hoechst 33342. (K) Bar plot reporting the average cross-sectional area in $\mu \mathrm{m}^{2}$ (wt LFD $n=8$; wt $\mathrm{HFD}$ $n=6 ; m d x$ LFD $n=6 ; m d x$ HFD $n=6$ ). Statistical significance was estimated by two-way ANOVA. (L) Immunoblot of TA lysates from wt and $m d x$ mice fed with LFD and HFD. Samples were probed with anti-pmTOR (Ser2448), anti-pRPS6 (Ser240/244), anti-mTOR, anti-RPS6, and anti-vinculin antibodies. (M) Bar plot showing the densitometric analysis of the phosphorylation level of mTOR (posho-mTOR). (N) Bar plot showing the densitometric analysis of the phosphorylation level of RPS6 (posho-RPS6). Statistical significance was estimated by two-way ANOVA. (0) Quantitative PCR of Igf1 in fibro/adipogenic progenitors from wt and mdx mice fed with LFD or HFD (wt LFD $n=3 ;$ wt HFD $n=4 ; m d x$ LFD $n=5 ; m d x$ HFD $n=7$ ). Statistical significance was estimated by two-way ANOVA. All data are represented as mean \pm SEM and statistical significance is defined as ${ }^{*} P<0.05 ;{ }^{*} P<0.01 ;{ }^{* *} P<0.001$. 
A

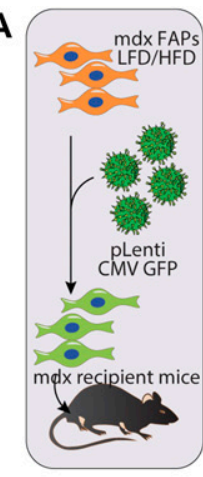

B

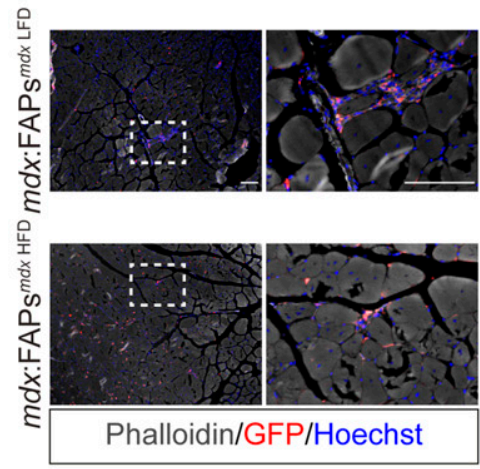

C
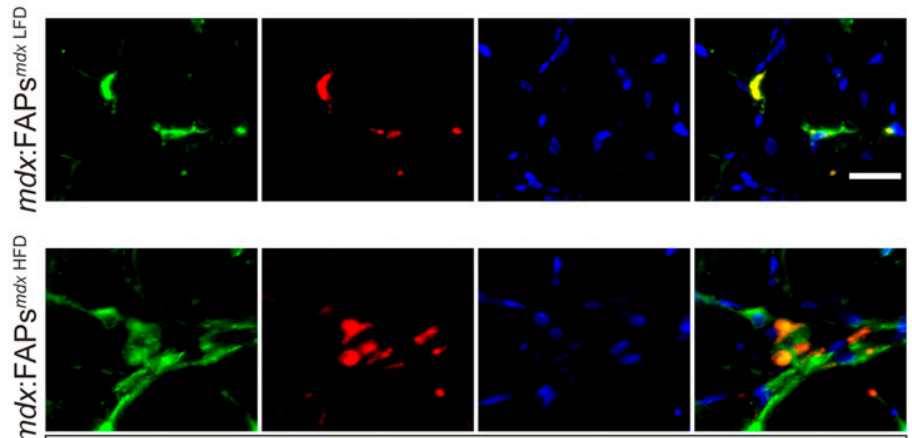

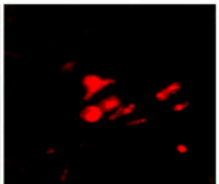

PDGFRa/GFP/Hoechst

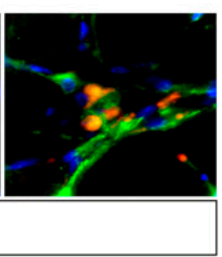

D

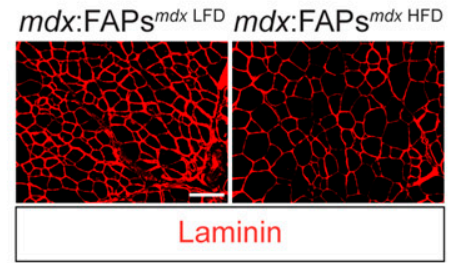

E

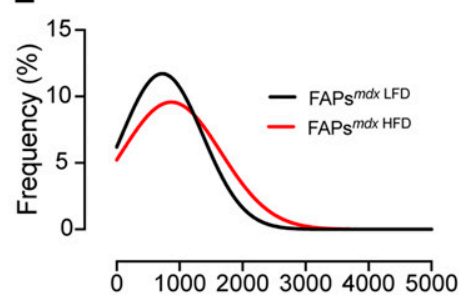

$F$

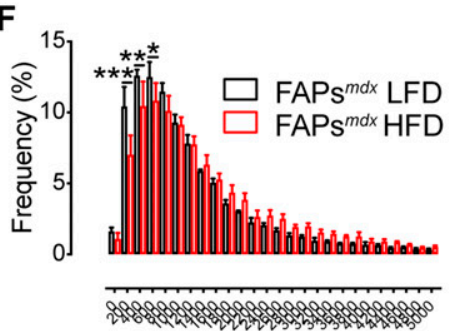

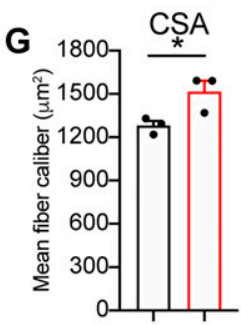

Figure 6. Fibro/adipogenic progenitors (FAPs) mediate the beneficial effects of high-fat diet (HFD).

(A) Workflow describing the FAP transduction and the transplantation strategies. FAPs were transplanted in 2 -mo-old $m d x$ recipient mice. FAPs (1.0 $\times 10^{5}$ cells) were resuspended in $100 \mu \mathrm{l}$ of $1 \times$ PBS and injected in the TA of mdx recipient mice. (B) After $15 \mathrm{~d}$ from transplantation, GFP-positive cells (red) were found in the muscle interstitium (10× and 40× magnification; scale bar, $100 \mu \mathrm{m})(n=3)$. Fibers (grey) were probed using phalloidin-488. (C) Representative immunofluorescence, showing that GFP-positive cells co-express the FAP distinctive marker PDGFR $\alpha(n=3)$. Nuclei (blue) were revealed with Hoechst 33342 . (D) Dystrophic ( $m d x)$ TA muscles receiving FAPS from LFD- and HFD-treated $m d x$ mice were stained with anti-laminin antibodies to reveal the fiber outlines. (E) Bar plot reporting the average percentage of the frequency distribution of the myofiber areas $\left(\mu \mathrm{m}^{2}\right)$ in each experimental condition $(n=3)$. $(\mathbf{E}, \mathbf{F})$ Curves, inferred on the basis of the cross-sectional area distribution in (E), showing the shift toward higher fiber calibers upon transplantation of $m d x$ FAPs from mice fed with HFD (red line). (G) Bar plot reporting the average $\mathrm{cross}$-sectional area in $\mu \mathrm{m}^{2}$ ( $n=$ 3). Statistical significance was estimated by $t$ test. All data are represented as mean \pm SEM and statistical significance is defined as ${ }^{\star} P<0.05 ;{ }^{\star \star} P<0.01 ;{ }^{* \star \star} P<0.001$.

enhancing the MuSCs myogenic activity. Remarkably, HFD-reprogrammed FAPs are able to promote the diet-dependent amelioration of the dystrophic phenotype. In addition, ex vivo experiments suggest that metabolic reprogramming may potentially decrease FAP pathological contribution to fibrotic scar and fat infiltrate formation in the late stages of the dystrophic disease.

Recently, ApoE deficiency was shown to have catastrophic effects in dystrophic mice under HFD (Milad et al, 2017), suggesting that the local and systemic effects of dietary interventions should be carefully evaluated in preclinical animal studies. Further exploration of the proteomic resource reported here may aid in the discovery of additional mechanisms connecting metabolism to FAP biology and muscular dystrophy.

The results presented here offer a proof of principle that metabolic reprogramming of muscle progenitor cells by a HFD regimen can have a positive effect on muscle regeneration in a dystrophy mouse model. Whether these findings can be extended to dystrophy patients and whether it can be considered a viable support strategy remain to be established.

\section{Materials and Methods}

The full list of key materials, reagents, animal strains, and software used in this work is reported in Table 1.

\section{Mouse models}

C57BL/6J (RRID:IMSR_JAX:000664) and C57BL10ScSn-Dmd ${ }^{m d x} / J$ mice (RRID:IMSR_JAX:001801) (referred to as wt and $m d x$, respectively) were purchased from the Jackson Laboratories. In our study, sexes were equally balanced between genotypes. Young (45- and 49- $d$ old) wt and $m d x$ mice were used in this work. Mice were bred and maintained according to the standard facility procedures. All experimental studies were conducted according to the rules of good animal experimentation I.A.C.U.C. n 432 of March 12, 2006, and under ethical approval released on December 11, 2012, from the Italian Ministry of Health, protocol \#20/01-D.

\section{Murine primary cells}

FAPs and MuSCs were isolated from the hind limbs of male and female wt and $m d x$ mice. Freshly sorted FAPs were resuspended in FAPs-GM consisting of DMEM GlutaMAX (25 mM Glc) supplemented with 20\% FBS, $10 \mathrm{mM}$ Hepes, 1 mM sodium pyruvate, and $100 \mathrm{U} / \mathrm{ml}$ penicillin/streptomycin (P/S). Freshly purified MuSCS growth medium (MuSCS-GM) consisting of DMEM GlutaMAX (25 mM Glc) supplemented with 20\% FBS, 10\% donor horse serum, 2\% chicken embryo extract, $10 \mathrm{mM}$ Hepes, $1 \mathrm{mM}$ sodium pyruvate, and $100 \mathrm{U} / \mathrm{ml} \mathrm{P} / \mathrm{S}$. The cells were cultivated at $37^{\circ} \mathrm{C}$ in $5 \% \mathrm{CO}_{2}$. 


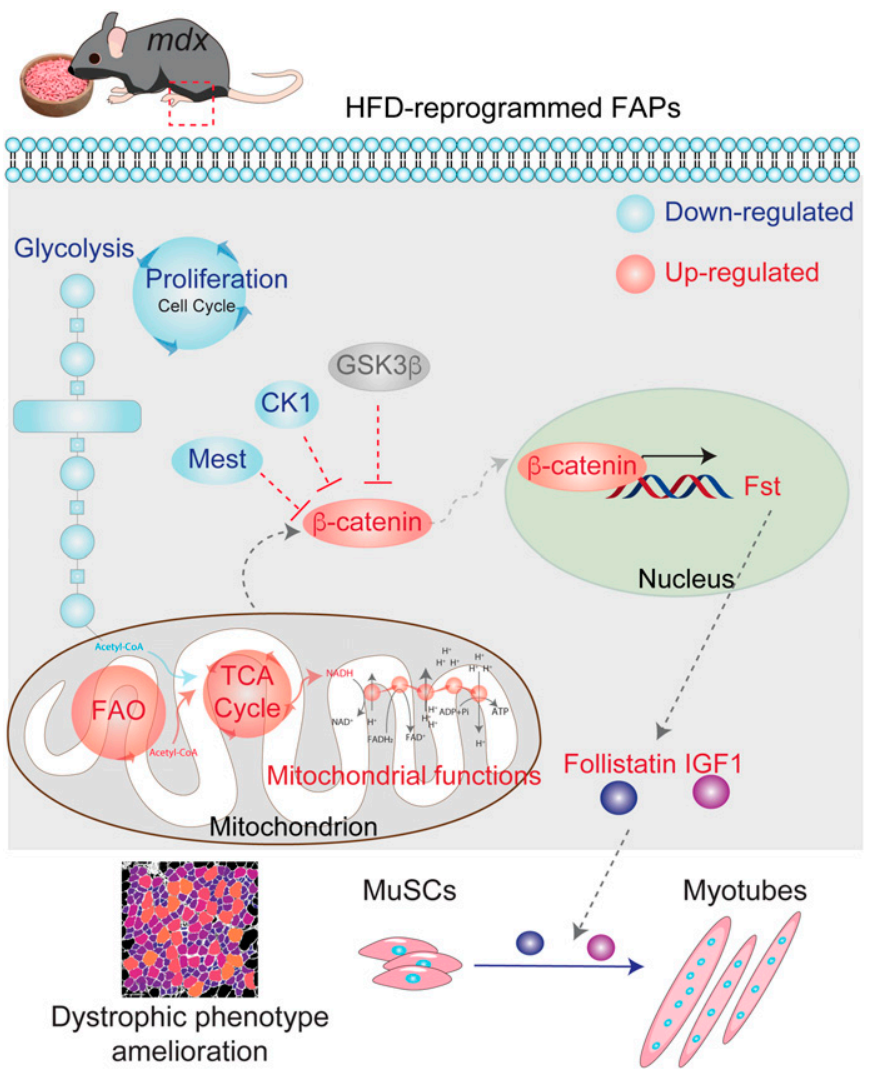

Figure 7. Working model proposal of the beneficial effects of high-diet in the dystrophic milieu.

A short-term high-fat diet (HFD) regimen provides a beneficial metabolic reprogramming of skeletal muscle interstitial fibro/adipogenic progenitors, dramatically affected in Duchenne muscular dystrophy. HFD restores the proper metabolic signature of dystrophic fibro/adipogenic progenitors, fueling mitochondrial pathways of fatty acid oxidation and tricarboxylic acid cycle and modulating the glycolytic flux. From the molecular point of view, $\beta$-catenin is a crucial hub that modulates muscle stem cells behavior. $\beta$-catenin inhibitors casein kinase (CK1) and MEST are repressed by HFD. The inhibition of glycogen synthase kinase 3 beta (GSK-3 $\beta$ ) activates the $\beta$-catenin signaling in turn modulating follistatin (Fst) expression Fst, in concert with IGF1, is released to sustain the differentiation of muscle satellite cells and myotube hypertrophy. The beneficial effects of HFD lead to the amelioration of the dystrophic phenotype.

\section{Diet treatment}

At the weaning day, wt and $m d x$ littermate were individually housed and randomly assigned to the LFD or HFD group with free access to food and water. In both groups, sexes were equally balanced between genotypes. Mice were fed with LFD (containing $11 \mathrm{kcal} \%$ fat, Research Diet) and HFD (containing 58 kcal\% fat, Research Diet D12331) for 4 wk (28 d). Body weight, food, and water intake were monitored every $2 \mathrm{~d}$. Although LFD and HFD differed in their fat content, both diets are isocaloric. Brain, spleen, liver, adipose tissue depots, and hearth were weighted and explanted from each mouse, snap-frozen in liquid nitrogen, and stored at $-80^{\circ} \mathrm{C}$ for further investigations.

\section{Isolation of MuSCs and FAPs from skeletal muscles}

Hind limbs were surgically removed and then minced in HBSS (GIBCO) supplemented with $100 \mathrm{U} / \mathrm{ml} \mathrm{P} / \mathrm{S}$ (Roche) and 0.2\% BSA
(Applichem). For each mouse, the homogeneous muscle tissue preparation was enzymatically digested in $2 \mu \mathrm{g} / \mu \mathrm{l}$ collagenase A, $2.4 \mathrm{U} / \mathrm{ml}$ dispase II, and $10 \mu \mathrm{g} / \mathrm{ml}$ DNase I (Roche) in Dulbecco's phosphate-buffered saline (BioWest) $\mathrm{w} /$ calcium and magnesium. The enzymatic digestion was performed for $1 \mathrm{~h}$ at $37^{\circ} \mathrm{C}$ with gentle shaking. The digested tissues underwent consecutive filtration through 100, 70, and $40 \mu \mathrm{m}$ cell strainers (Corning). Before each filtration step, the cells were centrifuged at $700 \mathrm{~g}$ for $10 \mathrm{~min}$ at $4^{\circ} \mathrm{C}$ and then resuspended in fresh HBSS. Red blood cells were lysed in RBC lysis buffer (Santa Cruz). Freshly isolated muscle mononuclear cells were resuspended in Magnetic beads buffer $(0.5 \%$ BSA and 2 mM EDTA in $1 \times$ PBS) and filtered through a 30- $\mu$ m PreSeparation Filter (Miltenyi) to remove large particles from the single cell suspension. The whole cell suspension underwent subsequential incubations with the microbead-conjugated antibodies used for the magnetic sorting. CD $45^{+}$immune cells and $\mathrm{CD} 1^{+}$endothelial cells were collected through the consecutive incubation with the anti-CD45 and anti-CD31 antibodies (Miltenyi). Lineage negative (Lin-) cells were incubated with anti- $\alpha 7$-integrin antibodies (Miltenyi) and MuSCs selected as Lin-/ $\alpha 7$-int+ cells. Last, Lin-/ $\alpha 7$-int+ cells were incubated with anti-Sca-1 antibodies (Miltenyi) and FAPs selected as Lin-/ $\alpha 7-$ int+/Sca- $1+$. The sorting procedures and the labelling procedures with the microbeadconjugated antibodies were performed according to the manufacturer's instructions.

\section{Lentiviral transduction and FAP transplantation}

FAPs were isolated from 49- $d$-old $m d x$ mice fed with LFD or HFD ( $n=$ 3) and transduced through spinoculation (Judson et al, 2018) with one MOI of pLenti CMV GFP Hygro (Addgene). Briefly, $1.0 \times 10^{5}$ cells were plated in serum-free medium (DMEM GlutaMax) in six-well plate and one $\mathrm{MOI}$ of virus was administered dropwise. The plate was centrifuged using a swinger rotor adapter for $5 \mathrm{~min}$ at 3,200 $\mathrm{g}$ at $25^{\circ} \mathrm{C}$, then for $1 \mathrm{~h}$ at $2,500 \mathrm{~g}$ at $25^{\circ} \mathrm{C}$. Virus-containing medium was discarded and the cells were washed twice with serum-free medium and scraped using a soft-gummy blade cell scraper. The cells were separated through centrifugation and resuspended in $100 \mu \mathrm{l}$ $1 \times$ PBS $\mathrm{W} / \mathrm{Ca}^{2+}$ and $\mathrm{Mg}^{2+}$. The cells were inoculated in TA muscles of anesthetized mice. TA muscles were collected after $15 \mathrm{~d}$.

\section{FAP culture and differentiation}

Freshly sorted FAPs were resuspended in FAPs-GM consisting of high-glucose (25 mM) DMEM GlutaMAX supplemented with 20\% FBS, $10 \mathrm{mM}$ Hepes, $1 \mathrm{mM}$ sodium pyruvate, and $100 \mathrm{U} / \mathrm{ml} \mathrm{P} / \mathrm{S}$ and plated in 96-well plates at a cell density of $3.0 \times 10^{4}$ cells $/ \mathrm{cm}^{2}$. After $3 / 4 \mathrm{~d}$, the FAP-GM was fully refreshed and cells cultured for two additional days before the induction of the adipogenic differentiation. The adipogenic differentiation was induced incubating FAPs with the adipocyte differentiation medium (ADM: FAPs-GM supplemented with $1 \mu \mathrm{g} / \mathrm{ml}$ insulin, $0.5 \mathrm{mM}$ 3-isobutyl-1-methylxanthine, and $1 \mu \mathrm{M}$ dexamethasone) for $3 \mathrm{~d}$ followed by two additional days in adipocyte maintenance medium (AMM: FAP-GM supplemented with $1 \mu \mathrm{g} / \mathrm{ml}$ insulin). Unstimulated cells were maintained in fresh FAPGM. For allowing spontaneous FAP growth and adipogenesis, the cells were plated in 96-well plates at a density of $7.5 \times 10^{3}$ cells $/ \mathrm{cm}^{2}$ 
Table 1. List of key materials, reagents, animal strains, and software.

\begin{tabular}{|c|c|c|}
\hline Reagent or resource & Source & Identifier \\
\hline \multicolumn{3}{|l|}{ Antibodies } \\
\hline Mouse mAb anti-vinculin (SPM227) & Abcam & Cat. no. ab18058; RRID:AB_444215 \\
\hline Total OXPHOS Rodent WB antibody cocktail & Abcam & Cat. no. ab110413; RRID:AB_2629281 \\
\hline Goat polyAb anti-mouse IgG $(\mathrm{H}+\mathrm{L})$-HRP & Bio-Rad & Cat. no. 1721011; RRID:AB_11125936 \\
\hline Goat polyAb anti-rabbit IgG $(\mathrm{H}+\mathrm{L})-\mathrm{HRP}$ & Bio-Rad & Cat. no. 1706515; RRID:AB_11125142 \\
\hline Rabbit mAb Ki67 (D3B5) & Cell Signaling Technology (CST) & Cat. no. 9129; RRID:AB_2687446 \\
\hline $\begin{array}{l}\text { Rabbit mAb anti-non-phospho active } \beta \text {-catenin (Ser33/ } \\
\text { 37/Thr41) (D13A1) }\end{array}$ & CST & Cat. no. 8814; RRID:AB_11127203 \\
\hline Rabbit mAb anti-PKM2 (D78A4) XP & CST & Cat. no. 4053; RRID:AB_1904096 \\
\hline Rabbit mAb anti-PPARy (81B8) & CST & Cat. no. 2243; RRID:AB_823598 \\
\hline Rabbit polyAb anti-perilipin1 (D418) & CST & Cat. no. 3470; RRID:AB_2167268 \\
\hline MYH1E antibody (MF 20) & Developmental Studies Hybridoma Bank & Cat. no. MF 20; RRID:AB_2147781 \\
\hline PAX7 antibody & Developmental Studies Hybridoma Bank & Cat. no. PAX7; RRID:AB_2299243 \\
\hline $\begin{array}{l}\mathrm{F}\left(\mathrm{ab}^{\prime}\right) 2 \text {-goat anti-mouse IgG }(\mathrm{H}+\mathrm{L}) \text { cross-adsorbed } \\
\text { secondary antibody, Alexa Fluor } 555\end{array}$ & Invitrogen & Cat. no. A-21425; RRID:AB_2535846 \\
\hline $\begin{array}{l}\text { Goat anti-mouse IgG }(\mathrm{H}+\mathrm{L}) \text { cross-adsorbed secondary } \\
\text { antibody, Alexa Fluor } 488\end{array}$ & Invitrogen & Cat. no. A-11001; RRID:AB_2534069 \\
\hline Alexa Fluor 488 phalloidin & Invitrogen & Cat. no. A12379 \\
\hline a7-Integrin MicroBeads, mouse & Miltenyi Biotec & Cat. no. 130-104-261 \\
\hline CD31 MicroBeads, mouse & Miltenyi Biotec & Cat. no. $130-097-418$ \\
\hline CD45 MicroBeads, mouse & Miltenyi Biotec & Cat. no. 130-052-301 \\
\hline Sca1 MicroBeads, mouse & Miltenyi Biotec & Cat. no. 130-106-641 \\
\hline Goat polyAb anti-PDGFRa & R\&D Systems & Cat. no. AF1062; RRID:AB_2236897 \\
\hline Rabbit polyAb MyoD (M-318) & Santa Cruz Biotechnology & Cat. no. sc-760; RRID:AB_2148870 \\
\hline Rabbit polyAb GFP antibody (FL) & Santa Cruz Biotechnology & Cat. no. Sc-8334; RRID:AB_641123 \\
\hline Rabbit polyAb anti-Lamin & Sigma-Aldrich & Cat. no. L9393; RRID:AB_477163 \\
\hline $\begin{array}{l}\text { Donkey anti-goat IgG }(\mathrm{H}+\mathrm{L}) \text {, multi-species SP ads-Alexa } \\
\text { Fluor } 488\end{array}$ & SouthernBiotech & Cat. no. $6425-30$ \\
\hline Goat anti-mouse $\lg G(\mathrm{H}+\mathrm{L})$, human ads-Alexa Fluor 555 & SouthernBiotech & Cat. no. 1031-32 \\
\hline $\begin{array}{l}\text { Goat anti-rabbit IgG(H+L), mouse/human ads-Alexa } \\
\text { Fluor } 488\end{array}$ & SouthernBiotech & Cat. no. $4050-30$ \\
\hline Goat $F\left(a b^{\prime}\right) 2$ anti-mouse Ig, human ads-Alexa Fluor 647 & SouthernBiotech & Cat. no. 1012-31 \\
\hline CD140a (PDGFRA) monoclonal antibody (APA5), APC & Thermo Fisher Scientific & Cat. no. 17-1401-81; RRID:AB_529482 \\
\hline ITGA7 monoclonal antibody (334908), APC & Thermo Fisher Scientific & Cat. no. MA5-23555; RRID:AB_2607368 \\
\hline Pax7 antibody (SPM613) (FITC) & Novus Biologicals & Cat. no. NBP2-47923F \\
\hline \multicolumn{3}{|l|}{ Chemicals, peptides and recombinant proteins } \\
\hline pLenti CMV GFP Hygro (656-4) & Addgene & Cat. no. 17446-LVC \\
\hline Seahorse XF Base Medium & Agilent Technologies & Cat. no. $102353-100$ \\
\hline Seahorse XF96 Flux Pack & Agilent Technologies & Cat. no. 102416-100 \\
\hline Bovin Serum Albumin (BSA) Fraction $V$ & Applichem & Cat. no. A1391 \\
\hline EDTA & Applichem & Cat. no. A5097 \\
\hline Killik, embedding medium for criostate blue & Bio-Optica & Cat. no. 059801 \\
\hline Clarity Western ECL Blotting Substrates & Bio-Rad & Cat. no. $10070-5061$ \\
\hline Criterion TGX Gradient gel 4-15\% & Bio-Rad & Cat. no. 5671085 \\
\hline
\end{tabular}


Table 1. Continued

\begin{tabular}{|c|c|c|}
\hline Criterion TGX Gradient gel 4-20\% & Bio-Rad & Cat. no. 5671095 \\
\hline Mini-PROTEAN TGX gel 4-15\% & Bio-Rad & Cat. no. 4561086 \\
\hline Mini-PROTEAN TGX gel 4-20\% & Bio-Rad & Cat. no. 4561096 \\
\hline Trans-Blot Turbo Midi Nitrocellulose Membrane & Bio-Rad & Cat. no. 1704157 \\
\hline Trans-Blot Turbo Mini Nitrocellulose Membrane & Bio-Rad & Cat. no. 1704156 \\
\hline DPBS $1 \times \mathrm{w} / \mathrm{Ca}^{2+}, \mathrm{Mg}^{2+}$ & BioWest & Cat. no. L0625-500 \\
\hline DPBS $1 \times \mathrm{w} / \mathrm{o} \mathrm{Ca}^{2+}, \mathrm{Mg}^{2+}$ & BioWest & Cat. no. L0615-500 \\
\hline Cell-Tak Cell and Tissue adhesive & Corning & Cat. no. 354240 \\
\hline Falcon $100-\mu \mathrm{m}$ cell strainer & Corning & Cat. no. 352360 \\
\hline Falcon $40-\mu \mathrm{m}$ cell strainer & Corning & Cat. no. 352340 \\
\hline Falcon $70-\mu \mathrm{m}$ cell strainer & Corning & Cat. no. 352350 \\
\hline Matrigel basement membrane mix & Corning & Cat. no. 356234 \\
\hline Eukitt mounting medium & Electron Microscopy Sciences & Cat. no. 15320 \\
\hline Donor horse serum & Euroclone & Cat. no. ECS0090D \\
\hline Fetal bovin serum (FBS) & Euroclone & Cat. no. ECS0180L \\
\hline Goat serum & Euroclone & Cat. no. ECS0200D \\
\hline Non-fat dried milk & Euroclone & Cat. no. EMR180500 \\
\hline Trypsin-EDTA $0.05 \%$ w/phenol red & Euroclone & Cat. no. ECM0920D \\
\hline Dulbecco's Modified Eagle's Medium (DMEM) GlutaMAX & GIBCO & Cat. no. 61965-026 \\
\hline $\begin{array}{l}\text { Dulbecco's Modified Eagle's Medium (DMEM) no } \\
\text { glucose, no glutamine, phenol red }\end{array}$ & GIBCO & Cat. no. A1443001 \\
\hline GlutaMAX Supplement & GIBCO & Cat. no. 35050038 \\
\hline $\mathrm{HBSS} \mathrm{w} / \mathrm{O} \mathrm{Ca}^{2+}, \mathrm{Mg}^{2+}$ & GIBCO & Cat. no. 14170112 \\
\hline Penicillin-streptomycin (P/S) 10,000 U/ml & GIBCO & Cat. no. 15140122 \\
\hline RPMI 1640 & GIBCO & Cat. no. 21875-091 \\
\hline Cell culture microplate, black & Greiner Bio One & Cat. no. 655090 \\
\hline Hoecsth 33342 & Invitrogen & Cat. no. H3570 \\
\hline MitoTracker Green FM & Invitrogen & Cat. no. M7514 \\
\hline MitoTracker Red CMXRos & Invitrogen & Cat. no. M7512 \\
\hline Novex NuPAGE Sample Reducing Agent (10x) & Invitrogen & Cat. no. NP0009 \\
\hline NUPAGE LDS Sample Buffer (4×) & Invitrogen & Cat. no. NP0007 \\
\hline TRIzol & Invitrogen & Cat. no. 15596026 \\
\hline Sodium fluoride (NaF) & Millipore & Cat. no. 67414 \\
\hline MS columns & Miltenyi Biotec & Cat. no. 130-042-201 \\
\hline Pre-separation filters $(30 \mu \mathrm{m})$ & Miltenyi Biotec & Cat. no. $130-041-407$ \\
\hline Histo-Clear Solution & National diagnostics & Cat. no. HS-200 \\
\hline Ortovanadate & New England Biolabs & Cat. no. P0758S \\
\hline $11 \mathrm{kcal} \%$ fat w/sucrose Surwit Diet (LFD) & Research diet & \\
\hline 58 kcal\% fat w/sucrose Surwit Diet (HFD) & Research diet & \\
\hline Cyto-Grow & Resnova & Cat. no. TGM-9001-A \\
\hline Collagenase A & Roche & Cat. no. 11088793001 \\
\hline Dispase II & Roche & Cat. no. 04942078001 \\
\hline DNase I & Roche & Cat. no. 11284932001 \\
\hline Paraformaldehyde solution (PFA) $4 \%$ in PBS & Santa Cruz & Cat. no. sc-281692 \\
\hline
\end{tabular}

(Continued on following page) 
Life Science Alliance

\section{Table 1. Continued}

\begin{tabular}{|c|c|c|}
\hline RBC Lysis buffer 10x & Santa Cruz Biotechnology & Cat. no. sc-296258 \\
\hline LY2090314 & Selleckchem & Cat. no. S7063 \\
\hline Chicken embryo extract & Seralab & Cat. no. CE-650-J \\
\hline 3-Isobutyl-1-methylxanthine (IBMX) & Sigma-Aldrich & Cat. no. 15879 \\
\hline Antimycin & Sigma-Aldrich & Cat. no. A8674 \\
\hline D-Glucose & Sigma-Aldrich & Cat. no. G8270 \\
\hline Dexamethasone & Sigma-Aldrich & Cat. no. D4902 \\
\hline Dimethyl sulfoxide (DMSO) Hybri-Max & Sigma-Aldrich & Cat. no. D2650 \\
\hline EGTA & Sigma-Aldrich & Cat. no. E3889 \\
\hline Eosin & Sigma-Aldrich & Cat. no. E4009 \\
\hline Hepes $1 \mathrm{M}$ & Sigma-Aldrich & Cat. no. $\mathrm{H} 0887$ \\
\hline Inhibitor phosphatase mixture II & Sigma-Aldrich & Cat. no. P5726 \\
\hline Inhibitor phosphatase mixture III & Sigma-Aldrich & Cat. no. P0044 \\
\hline Insulin solution human & Sigma-Aldrich & Cat. no. 19278 \\
\hline Magnesium chloride & Sigma-Aldrich & Cat. no. M8266 \\
\hline Oil Red O & Sigma-Aldrich & Cat. no. 00625 \\
\hline Oleic acid & Sigma-Aldrich & Cat. no. 01008 \\
\hline Oligomycin & Sigma-Aldrich & Cat. no. 04876 \\
\hline Sodium pyruvate $100 \mathrm{mM}$ & Sigma-Aldrich & Cat. no. S8636 \\
\hline TERGITOL Solution NP-40 & Sigma-Aldrich & Cat. no. NP40S \\
\hline Triton $\mathrm{X}-100$ & Sigma-Aldrich & Cat. no. T9284 \\
\hline Trizma base & Sigma-Aldrich & Cat. no. T1503 \\
\hline Tween 20 & Sigma-Aldrich & Cat. no. P1379 \\
\hline \multicolumn{3}{|l|}{ Critical commercial assays } \\
\hline Click-iT EdU Alexa Fluor 488 Imaging Kit & Invitrogen & Cat. no. C10337 \\
\hline CellTiter-Glo luminescent cell viability assay & Promega & Cat. no. G7571 \\
\hline Custom mouse follistatin ELISA & RayBiotech & Cat. no. ELM-FST-1 \\
\hline In situ cell death detection lit, TMR red & Sigma-Aldrich & Cat. no. 12156792910 \\
\hline \multicolumn{3}{|l|}{ Deposited data } \\
\hline $\begin{array}{l}\text { Proteome of wt and } m d x \text { FAPs from mice fed with LFD } \\
\text { and HFD }\end{array}$ & This paper & $\mathrm{N} / \mathrm{A}$ \\
\hline $\begin{array}{l}\text { Proteome of wt and } m d x \text { MuSCs from mice fed with LFD } \\
\text { and HFD }\end{array}$ & This paper & $\mathrm{N} / \mathrm{A}$ \\
\hline
\end{tabular}

(Continued on following page) 
Table 1. Continued

\begin{tabular}{|c|c|c|}
\hline Proteome of wt and $m d x$ FAPs & Marinkovic et al (2019) & N/A \\
\hline \multicolumn{3}{|c|}{ Experimental models: organisms/strains } \\
\hline Mouse $\mathrm{C} 57 \mathrm{BL} / 6 \mathrm{~J}$ & Jaxmice & $\begin{array}{l}\text { Cat. no. JAX:000664; RRID:IMSR_JAX: } \\
000664\end{array}$ \\
\hline Mouse C57BL10ScSn-Dmdmdx/J & Jaxmice & $\begin{array}{l}\text { Cat. no. JAX:001801; RRID:IMSR_JAX: } \\
001801\end{array}$ \\
\hline \multicolumn{3}{|l|}{ Software and algorithms } \\
\hline Illustrator CC 2017 & $\begin{array}{l}\text { Adobe; http://www.adobe.com/products/ } \\
\text { illustrator.html }\end{array}$ & RRID:SCR_010279 \\
\hline CytExpert software & Beckman Coulter & RRID:SCR_017217 \\
\hline Photoshop CC 2017 & $\begin{array}{l}\text { Adobe; https://www.adobe.com/products/ } \\
\text { photoshop.html }\end{array}$ & RRID:SCR_014199 \\
\hline GraphPad Prism & GraphPad Software; http://www.graphpad.com/ & RRID:SCR_002798 \\
\hline Cytoscape 3.7 .1 & http://cytoscape.org; Shannon et al (2003) & RRID:SCR_003032 \\
\hline Fiji & http://fiji.sc; Schindelin et al (2012) & RRID:SCR_002285 \\
\hline MaxQuant 1.5.3.6 & $\begin{array}{l}\text { http://www.biochem.mpg.de/5111795/maxquant; Cox } \\
\text { and Mann (2008) }\end{array}$ & RRID:SCR_014485 \\
\hline Perseus 1.6.2.3 & $\begin{array}{l}\text { http://www.perseus-framework.org; Tyanova et al } \\
\text { (2016) }\end{array}$ & RRID:SCR_015753 \\
\hline Seahorse Wave Desktop software & $\begin{array}{l}\text { Agilent; http://www.agilent.com/en-us/products/cell- } \\
\text { analysis-(seahorse)/software-download-for-wave- } \\
\text { desktop }\end{array}$ & RRID:SCR_014526 \\
\hline
\end{tabular}

in FAPs-GM. The medium was refreshed every $2 \mathrm{~d}$ for $20 \mathrm{~d}$. Metabolic perturbations were performed during the differentiation period by supplementing ADM and AMM with $250 \mu \mathrm{M}$ of 2-DG or by replacing Glu with Gal. Palmitate and oleate were dissolved in ethanol at 200 $\mathrm{mM}$. Each fatty acid was diluted separately at $3 \mathrm{mM}$ in a solution of $10 \% \mathrm{BSA}$ in PBS $1 \times \mathrm{W} / \mathrm{Ca}^{2+}, \mathrm{Mg}^{2+}$, and incubated overnight at $37^{\circ} \mathrm{C}$ in gentle shaking. Solution were filtered through a syringe filter of 0.22 $\mu \mathrm{m}$. Separate stock solution were aliquoted and stored at $-20^{\circ} \mathrm{C}$. Freshly thawed aliquots were used for treatments. Pharmacological blockade of GSK3 $\beta$ was performed at the onset of the adipogenic differentiation, by supplementing ADM with 20 nM LY2090314. Further experiments involving the use of LY2090314 were performed using in vitro subcultured FAPs. In this case, FAPs were grown in Cyto-Grow (Resnova) by plating $2.5 \times 10^{3}$ cells $/ \mathrm{cm}^{2}$. The cells were not passaged more than once and routinely checked for their multipotency. Nearto-confluent cells were dethatched and cultured as described above in FAP-GM.

\section{MuSC culture and differentiation}

To reduce fibroblasts contamination, freshly sorted $w t$ and $m d x$ MuSCs were pre-plated for $2 \mathrm{~h}$ in pre-warmed MuSC Growth Medium (MuSCs-GM) consisting of high-glucose (25 mM) DMEM GlutaMAX supplemented with 20\% FBS, $10 \%$ donor horse serum, $2 \%$ chicken embryo extract, $10 \mathrm{mM}$ Hepes, $1 \mathrm{mM}$ sodium pyruvate, and $100 \mathrm{U} / \mathrm{ml}$ $\mathrm{P} / \mathrm{S}$. Pre-plated MuSCs were seeded in MuSC-GM at a cell density of $1.5 \times 10^{4}$ cells $/ \mathrm{cm}^{2}$ in Matrigel-coated 96 -well plates. After $3 \mathrm{~d}$, MuSCGM was fully refreshed and cells cultured for three additional days allowing spontaneous myotube formation. Metabolic perturbations were carried out in the last $3 \mathrm{~d}$ of MuSC differentiation by incubating cells with the MuSC-GM supplemented with $250 \mu \mathrm{M}$ of 2-DG or by replacing Glu with Gal.

\section{Follistatin (FST) ELISA assay}

The Follistatin concentration in FAP-derived culture supernatants was assessed using Custom Mouse Follistatin ELISA Kit (RayBiotech) according to the manufacturer's instructions. FST standard curve was prepared through serial dilution. FAP CM were diluted 1:4 with Assay Diluent. $100 \mu \mathrm{l}$ of standard/samples were loaded in duplicate. Colorimetric assay was performed at $450 \mathrm{~nm}$.

\section{FAP-derived CM}

Freshly sorted FAPs from mice fed with LFD and HFD were plated in FAPs-GM at a density of $3.0 \times 10^{4}$ cells $/ \mathrm{cm}^{2}$. After $4 \mathrm{~d}$ of culture, $\mathrm{CM}$ were recovered, centrifuged at $3,000 \mathrm{~g}$ to remove cells, and stored at $-80^{\circ} \mathrm{C}$ before use. The control CM was prepared by incubating empty wells with the same medium. CM treatments were performed on 2-d cultured mdx MuSCs. Briefly, MuSC-GM was removed and replaced by FAP-derived CMs. Cells were fixed $3 \mathrm{~d}$ later.

\section{Proteome sample preparation}

Cells were lysed in sodium deoxycholate (SDC) lysis buffer containing 4\% (wt/vol) SDC, 100 mM Tris-HCl (pH 8.5). Proteome preparation was done using the in StageTip (iST) method (Kulak et al, 2014). Samples 
were separated by HPLC in a single run (without pre-fractionations) and analyzed by MS.

\section{LC-MS/MS measurements}

The peptides were separated on a reverse-phase column $(50 \mathrm{~cm}$, packed in-house with 1.9- $\mu \mathrm{m}$ C18-Reprosil-AQ Pur reversed-phase beads) ( $\mathrm{Dr}$ Maisch $\mathrm{GmbH}$ ) over 120 min (single-run proteome analysis). Eluting peptides were electrosprayed and analyzed by tandem MS on a Q Exactive HF (Thermo Fischer Scientific) using higher-energy collisional dissociation (HCD)-based fragmentation, which was set to alternate between a full scan followed by up to five fragmentation scans.

\section{Proteome data processing}

Raw MS data were analyzed in the MaxQuant environment (Cox \& Mann, 2008), version 1.5.1.6, using the Andromeda engine for database search. MS/MS spectra were matched against the Mus Musculus UniProt FASTA database (September 2014), with an false discovery rate (FDR) of $<1 \%$ at the level of proteins, peptides, and modifications. Enzyme specificity was set to trypsin, allowing cleavage of $\mathrm{N}$-terminal to proline and between aspartic acid and proline. The search included cysteine carbamidomethylation as a fixed modification, and $\mathrm{N}$-terminal protein acetylation and oxidation of methionine. Label-free proteome analysis was performed in MaxQuant. Where possible, the identity of peptides present but not sequenced in a given run was obtained by transferring identifications across LC-MS runs ("match between runs"). Up to three missed cleavages were allowed for protease digestion and peptides had to be fully tryptic.

\section{Proteome bioinformatics data analysis}

Bioinformatics analysis was performed in the Perseus software environment (Tyanova et al, 2016). Statistical analysis of proteome was performed on logarithmized intensities for those values that were found to be quantified in any experimental condition. To identify significantly modulated proteins, missing values (proteins not identified or not quantified) were imputed by using a normal distribution. Then, we performed a $t$ test with a P-value cutoff of 0.05 and $\mathrm{SO}=0.1$. Categorical annotation was added in Perseus in the form of gene ontology (GO) biological process (GOBP), molecular function (GOMF), and cellular component (GOCC), and KEGG pathways and Keywords (extracted from Uniprot). We performed a 2D annotation enrichment analyses to identify statistically significant GO-terms, KEGG pathways, and Keywords enriched in mdx HFD FAPs (Cox \& Mann, 2008). Multiple hypothesis testing is controlled by using a Benjamini-Hochberg FDR threshold of 0.07. Then for each term, the corresponding $P$-value and score are assigned. Whereas a score near 1 indicates a positive enrichment, a score near -1 means a negative enrichment of the category. The complete proteome measurements for FAPs and MuSCs are collected in Tables S1 and S2, respectively.

\section{Network analysis}

This strategy has been previously developed and applied by our group (Sacco et al, 2012, 2016). Kinase-substrate relationships and physical interactions were extracted from SIGNOR and MINT, respectively (Zanzoni et al, 2002; Perfetto et al, 2015), and were mapped onto the complete human proteome in Cytoscape (Shannon et al, 2003). Then the network was first filtered to maintain only relationships between proteins that were significantly modulated in $m d x$ LFD FAPs compared with wt LFD FAPs and in $m d x$ HFD FAPs compared with $m d x$ LFD FAPS.

\section{Flow cytometry}

Immunophenotyping of Sca1+ FAPs a was performed by staining $4.0 \times$ $10^{5}$ cells with anti-CD140a (APC) (PDGFR $\alpha$ ) antibody for 30 min at $4^{\circ} \mathrm{C}$. Immunophenotyping of ITGA7+ MuSCs was performed by staining $4.0 \times$ $10^{5}$ cells with anti-ITGA7 (APC) antibody for $30 \mathrm{~min}$ at $4^{\circ} \mathrm{C}$. Subsequently, MuSCs were permeabilized with $0.3 \%$ Triton-X 100 in $1 \times$ PBS for 15 min at $4^{\circ} \mathrm{C}$. Permeabilized cells were incubated with anti-Pax7 (FITC) for $30 \mathrm{~min}$ at $4^{\circ} \mathrm{C}$. The cells were washed twice and analyzed using CytoFlex Cytometer (Beckman Coulter). Data were analyzed using the Cytexpert Software (Beckman Coulter).

Freshly purified wt and $m d x$ FAPs were resuspended in FAPs-GM and incubated in the absence/presence of $10 \mu \mathrm{M}$ carbonyl cyanidep-trifluoromethoxyphenylhydrazone (FCCP) for 30 min at $37^{\circ} \mathrm{C}$. After mitochondrial uncoupling, FAPs were incubated with $200 \mathrm{nM}$ of MitoTracker Red CMXRos or MitoTracker Green FM for 20 min. FAPs were washed twice with $1 \times$ PBS and fixed in 0.5\% PFA before flow cytometry analysis. For each sample, $10,000^{3}$ events were recorded using BD FACSCALIBUR (BD Biosciences).

\section{ATP measurement}

Freshly isolated FAPS were seeded at a cell density of $6 \times 10^{4}$ cells/ $\mathrm{cm}^{2}$ in 96 -well plates. After $20 \mathrm{~h}$, ATP content was evaluated with CellTiter-Glo Luminescent Viability Assay (Promega) according to the manufacturer's instructions. Briefly, CellTiter-Glo reagent was added at a ratio of 1:1 to cell culture medium and mixed for 2 min on an orbital shaker. Samples were transferred to a black-walled 96-well plate and luminescence was recorded through Victor Multilabel plate reader (PerkinElmer). Background consisted of FAP-GM incubated in the absence of FAPs and processed as described above. Data are presented as mean relative luminescence units.

\section{Bioenergetics analysis of FAPs and MuSCs}

Freshly isolated wt and $m d x$ FAPs/MuSCs were plated on Seahorse XFe96 Microplates (Agilent Technologies) at the density of $1.4 \times 10^{5}$ cells $/ \mathrm{cm}^{2}$ and cellular bioenergetics assessed $4 \mathrm{~d}$ later. Alternatively, subcultured FAPs were plated at the density of $7.0 \times 10^{4}$. After $24 \mathrm{~h}$, the cells were incubated in Cyto-Grow:RPMI in 1:1 ratio for $20 \mathrm{~h}$ to stimulate mitochondrial functionality before assessing the cellular bioenergetics. Cartridges were hydrated overnight with Seahorse XF Calibrant and incubated at $37^{\circ} \mathrm{C}$ in the absence of $\mathrm{CO}_{2}$. Calibrant was changed and refreshed $1 \mathrm{~h}$ before the assay. Mitochondrial stress test was performed according to Agilent's recommendations. Briefly, the cells were washed four times with Seahorse XF Base Medium supplemented with $10 \mathrm{mM}$ glucose, $1 \mathrm{mM}$ sodium pyruvate, and $2 \mathrm{mM}$ glutamine ( $\mathrm{pH} 7.4 \pm 0.01$ ). The cells were incubated at $37^{\circ} \mathrm{C}$ 
in the absence of $\mathrm{CO}_{2}$ for $1 \mathrm{~h}$. Mitochondrial inhibitors were sequentially injected at the following final concentrations: $1 \mu \mathrm{M}$ oligomycin, $1.5 \mu \mathrm{M}$ FCCP, and $1 \mu \mathrm{M} / 1 \mu \mathrm{M}$ Rotenone/Antimycin (Sigma-Aldrich).

\section{Immunoblot analysis}

FAPs were washed in PBS $1 \times$ and stored at $-80^{\circ} \mathrm{C}$ or directly lysed in ice-cold radio-immunoprecipitation assay lysis buffer $(150 \mathrm{mM} \mathrm{NaCl}$, $50 \mathrm{mM}$ Tris- $\mathrm{HCl}$, pH 7.5, 1\% Nonidet P-40, 1 mM EGTA, $5 \mathrm{mM} \mathrm{MgCl}$, and $0.1 \%$ SDS) supplemented with $1 \mathrm{mM}$ PMSF, $1 \mathrm{mM}$ ortovanadate, $1 \mathrm{mM}$ $\mathrm{NaF}$, protease inhibitor mixture 1×, inhibitor phosphatase mixture II 1×, and inhibitor phosphatase mixture III 1x. Protein lysates were separated at 15,500 $\mathrm{g}$ for $30 \mathrm{~min}$. The total protein concentration was determined using the Bradford reagent. Protein extracts were denatured and heated at $95^{\circ} \mathrm{C}$ for $10 \mathrm{~min}$ in NuPAGE LDS Sample Buffer that contained DTT as a reducing agent (NuPAGE Sample Reducing Agent). According to the needs, proteins were resolved using either 4-15\% or 4-20\% Bio-Rad Mini-PROTEAN TGX/CRITERION polyacrylamide gels. Proteins were transferred to Trans-Blot Turbo Mini or Midi Nitrocellulose Membranes using a Trans-Blot Turbo Transfer System (Bio-Rad), and the nonspecific binding membranes were saturated for $1 \mathrm{~h}$ at RT in blocking solution (5\% skimmed milk powder, 0.1\% Tween 20 in 1× TBS). Saturated membranes were incubated overnight with the specific primary antibodies diluted in blocking solution. Host-specific HRP-conjugated secondary antibodies were diluted in blocking solution and used for the detection of the primary antibodies. Chemiluminescent detection was performed using Clarity Western ECL Blotting Substrates (Bio-Rad) and the Las-3000 Imaging System (Fujifilm). Band densities were quantified using Fiji and normalized to the loading control. All antibodies were diluted according to the manufacturer's instructions.

\section{Real-time PCR}

Total RNA was extracted using TRIzol from TAs, diaphragms, and FAPs. Muscles were minced in short pieces and homogenized with a Dounce tissue grinder directly in TRIzol. Before resuspension, total RNA was precipitated overnight in the presence of $10 \mu$ g of glycogen. RNA concentration was assessed using NanoDrop Lite Spectrophotometer. Total RNA (ranging between 300 and 1,000 ng) was reverse-transcribed into CDNA with Primescript RT Reagent Kit. qPCR reactions were carried out with SYBR Premix Ex Taq (Tli RNaseH Plus) and performed in technical duplicates for each biological repeat. Each reaction mixture (final volume of $20 \mu \mathrm{l}$ ) contained 50 ng of cDNA. Relative expression was estimated by $2^{-\Delta c t}$ as described (Livak \& Schmittgen, 2001). To compare multiple runs, mRNA levels were determined by the $2^{-\Delta \Delta C t}$ method (Livak \& Schmittgen, 2001). Actb and Tuba1a were used as reference genes. The full primer list is reported in Table 2 .

\section{Histological analysis}

TA and diaphragm muscles were embedded in optimal cutting temperature medium and snap-frozen in liquid nitrogen. Embedded muscles were stored at $-80^{\circ} \mathrm{C}$ before cryosectioning.

Prior optimal cutting temperature embedding, for GFP immunolabelling in transplanted TAs, muscles were fixed in 4\% PFA for
$4 \mathrm{~h}$ at RT and incubated for $4 \mathrm{~h}$ in sucrose gradient solutions (10\%, $20 \%$ and $30 \%$ ). Muscles were transverse cryosectioned at $10 \mu \mathrm{m}$ using a Leica cryostat. Muscle sections were sequentially collected in different glass slides, thus adjacent sections were separated at least $100 \mu \mathrm{m}$ from each other. Cryosections were performed for the entire length of the muscles.

For the hematoxylin and eosin stain (H\&E), sections were fixed in 4\% PFA for 10 min at RT. Fixed sections were washed twice in 1× PBS and incubated in hematoxylin solution for $20 \mathrm{~min}$. Sections were rinsed for $10 \mathrm{~min}$ in tap water and washed in ultrapure water. Samples were incubated with the eosin solution for $1 \mathrm{~h}$. Finally, stained sections were ethanol-dehydrated, clarified with the HistoClear solution and mounted with the Eukitt mounting medium.

\section{Immunofluorescence}

Cells and sections were fixed in 2\% PFA for $15 \mathrm{~min}$ at RT. For immunocytochemistry fixed cells were washed twice in 1x PBS and incubated in permeabilization solution ( $0.5 \%$ Triton X-100 in 1× PBS) for $5 \mathrm{~min}$. Unspecific binding were saturated by incubating samples in blocking solution (10\% FBS and $0.1 \%$ Triton X-100 in $1 \times$ PBS) for $1 \mathrm{~h}$. The cells were incubated overnight at $4^{\circ} \mathrm{C}$ in gentle shaking in the presence of the primary antibodies. The cells were washed four times with $0.1 \%$ Triton X-100 in 1× PBS and incubated, for 30 min at RT, with host-specific secondary antibodies. Primary and secondary antibodies were diluted according to the manufacturer's recommendations in blocking solution. Finally, the cells were washed four times with $0.1 \%$ Triton X-100 in $1 \times$ PBS and counterstained for $5 \mathrm{~min}$ at RT with the Hoechst solution $(1 \mathrm{mg} / \mathrm{ml}$ Hoechst 33342 and $0.1 \%$ Triton X-100 in 1× PBS). Cells were washed twice with $1 \times$ PBS and stored at $4^{\circ} \mathrm{C}$.

For muscle section immunofluorescence, different protocols were adapted according to the antigen of interest. For laminin staining, fixed sections were washed twice in $1 \times$ PBS and permeabilized (0.3\% Triton X-100 in 1× PBS) for 30 min at RT. Permeabilized slices were incubated in blocking solution (10\% goat serum, $1 \%$ glycine, and $0.1 \%$ Triton X-100 in $1 \times$ PBS) for $1 \mathrm{~h}$ at RT. Blocked specimen were incubated overnight at $4^{\circ} \mathrm{C}$ in the presence of anti-laminin (Sigma-Aldrich) diluted 1:200 in blocking solution.

For the in situ detection of PDGFR $\alpha$, fixed and permeabilized sections were blocked for $30 \mathrm{~min}$ in protein block serum-free reagent (DAKO) and then incubated overnight with anti-PDGFR $\alpha$ diluted 1:80 in a carrier solution (4\% BSA in 1× PBS). After incubation with the primary antibodies, the sections were washed four times with $0.1 \%$ Triton X-100 in $1 \times$ PBS and incubated for 30 min at RT with host-specific secondary antibodies. Nuclei were revealed by incubating samples for 10 min with Hoechst solution. The sections were washed twice in $1 \times$ PBS and mounted with Aqua Poly/Mount mounting medium.

\section{TUNEL assay}

In situ detection of apoptotic nuclei was performed through the In Situ Cell Death Detection Kit, TMR red (Sigma-Aldrich). Cells were fixed with 2\% PFA for 20 min at RT and permeabilized with $0.5 \%$ Triton X-100 in 1× PBS for 5 min at RT. Positive controls were treated with DNAse I ( $3 \mathrm{U} / \mathrm{ml}$ in $50 \mathrm{mM}$ Tris $-\mathrm{HCl}, \mathrm{pH} 7.5$, and $1 \mathrm{mg} / \mathrm{ml} \mathrm{BSA}$ ) for 
Table 2. List of primers used in real time PCR experiments.

\begin{tabular}{|c|c|c|c|}
\hline Gene & ID & Forward primer & Reverse primer \\
\hline \multicolumn{4}{|c|}{ Primer list } \\
\hline Actb & 11461 & 5'-CACACCCGCCACCAGTTCGC-3' & 5'-TTGCACATGCCGGAGCCGTT-3' \\
\hline Clebpa & 12606 & 5'-GAGGGGAGGGACTTAGGTGT-3' & 5'-GGAGGTGCAAAAAGCAAGGG-3' \\
\hline Ctnnb1 & 12387 & 5'-TGGACCCTATGATGGAGCATG-3' & 5'-GGTCAGTATCAAACCAGGCCAG-3' \\
\hline $\lg 1$ & 16000 & 5'-GCTGGTGGATGCTCTTCAGT-3' & 5'-TCCGGAAGCAACACTCATCC-3' \\
\hline Mest & 17294 & 5'-GGCCATTGGATCCTATAAATCCGTA-3' & 5'-GGTAGTGGCTAATGTGGTCATCCAG-3' \\
\hline Myh3 & 12883 & 5'-TCGTCTCGCTTTGGCAA-3' & 5'-TGGTCGTAATCAGCAGCA-3' \\
\hline Pparg1 & 19016 & 5'-CGAGTGTGACGACAAGGTGA-3' & 5'-ACCGCTTCTTTCAAATCTTGTCTG-3' \\
\hline Pparg2 & 19016 & 5'-GCCTATGAGCACTTCACAAGAAAT-3' & 5'-GGAATGCGAGTGGTCTTCCA-3' \\
\hline Tuba1a & 22142 & 5'-AAGCAGCAACCATGCGTGA-3' & 5'-CCTCCCCCAATGGTCTTGTC-3' \\
\hline
\end{tabular}

10 min at RT. Negative controls were treated only with the enzyme buffer without transferase enzyme. All wells were incubated with 50 $\mu \mathrm{l}$ of reaction solution for $1 \mathrm{~h}$ at $37^{\circ} \mathrm{C}$ in humidified chamber. After labelling, the samples were washed with $1 \times$ PBS and counterstained with Hoechst 33342. Images were acquired as described in "Image acquisition."

\section{EdU incorporation assay}

For EdU labelling (Invitrogen), the cells were seeded at a concentration of $6.0 \times 10^{3}$ cells $/ \mathrm{cm}^{2}$ and incubated in the presence of 10 $\mu \mathrm{M}$ of 5-ethylnyl-2'-deoxyuridine (EdU) the day prior fixation. ClickiT reaction was performed according to the manufacturer's instructions.

\section{Oil Red 0 (ORO)}

Oil Red O (Sigma-Aldrich) stock solution was prepared according to the manufacturer's recommendations. Fixed and permeabilized cells were washed twice with $1 \times$ PBS and incubated for 10 min with filtered ORO working solution (3:2 ratio ORO:ultrapure water). Stained cells were washed twice for $10 \mathrm{~min}$ with $1 \times$ PBS and counterstained using Hoechst 33342. ORO-stained cells were acquired via fluorescence microscopy.

\section{Image acquisition}

Immunolabeled cells and section were acquired using the DMI6000B fluorescent microscope (Leica). Cell Micrographs were captured in unbiased fashion with the "matrix screener" mode, by acquiring nine non-overlapping field across the well.

Section micrographs were captured with DMI6000B and Nikon Eclipse TE300 microscopes (Nikon). At least three independent fields were captured in five nonadjacent sections for each mouse. Acquisition were performed at 40×, 20×, or 10× magnification.

Representative micrographs were captured using the confocal microscope Olympus IX-81 at 20x magnitude.

For bright-field microscopy, at least three non-overlapping micrographs of five independent $\mathrm{H} \& \mathrm{E}-$ stained sections were captured using a Zeiss Lab A1 AX10 microscope at the 20× magnification.

\section{Biochemical analysis}

Before sacrifice, a blood sample was collected from each mouse in SST microtainers by retro-orbital sampling. For biochemical clinical analysis, blood samples were incubated for $20 \mathrm{~min}$ at RT and sera separated at 15,500g for 7 min. Non-fasting glucose, triglycerides, cholesterol, creatine kinase, and creatinine were measured using the automatic analyzer Keylab (BPC BioSed Srl).

\section{Histological analysis}

Centrally nucleated myofibers were counted and scored manually using Fiji (Schindelin et al, 2012) by three independent collaborators in blind analysis. Results are expressed as ratio over the total myofibers.

CSA measurements of myofibers were conducted on lamininstained sections. The myofiber diameters were evaluated using a Fiji plug-in and data represented as average CSA and frequency distribution, for each mouse. For the pseudo-color representation of the CSA, images were processed with the ROI Color Coder macro using Fiji (http:/ /imagejdocu.tudor.lu/doku.php?id=macro:rol_color_coder) (Kopinke et al, 2017). The manual analysis of the images was performed in blind.

\section{Seahorse data processing}

OCR was normalized by estimating the number of cells for each experimental sample. Briefly, immediately after the assay, the cells were fixed with $2 \%$ PFA for 15 min. Nuclei were labelled with Hoechst 33342 and a representative field of each well was captured and automatically scored with CellProfiler (Carpenter et al, 2006). An estimation of the total nuclei number for each condition was calculated, correlating the field to the well areas. OCR values are expressed as pmol $\mathrm{O}_{2} / \mathrm{min} / 10^{3}$ cells. Mitochondria activity parameters were extracted with Agilent Wave Software (version 2.6.0).

\section{Cell differentiation measurements}

Adipogenic differentiation of FAPs was estimated in unbiased fashion using CellProfiler through a dedicated pipeline that 
recognize, for each captured field, the ORO-positive area (expressed in pixels). The modules of the pipeline were routinely adjusted in agreement with the image magnifications. The average ORO-positive area was normalized over the average number of cells per field. Alternatively, the percentage of adipogenic differentiation was evaluated by counting the number of adipocytes over the total cells in each field. Positive objects were manually scored using Fiji by two independent collaborators.

Adipocyte dimension was evaluated by measuring the area of each lipid droplet associated with each adipocyte. Briefly, three randomly and non-overlapping field of perilipin-stained adipocytes were acquired at 40× magnification. The area of each lipid droplet was delimited using the "Frehand selection" tool of Fiji. At least 15 adipocytes for each field were included in the analysis.

Myogenic differentiation of MuSCs was scored manually using Fiji, by calculating the fusion index. Fusion index was defined as the ratio between the number of nuclei included into $\mathrm{MyHC}$-expressing cells (containing at least three nuclei) over the number of nuclei for each field.

The percentage of EdU-positive cells was estimated in unbiased fashion through a dedicated pipeline using CellProfiler.

Before automated analysis, a supervised control of each CellProfiler module was performed to ensure the proper recognition of the markers of interest. The manual analysis of the images was performed in blind.

\section{Statistical analysis}

Data are represented as mean \pm SEM of at least three independent experimental samples unless otherwise mentioned. In addition, cell studies were further performed at least in technical duplicates. Statistical significance between two groups was estimated using the unpaired $t$ test assuming a two-tailed distribution. Multiple comparisons between three or more groups were performed using one-way or two-way ANOVA. Statistical significance is defined as * $P$ $<0.05 ;{ }^{* \star} P<0.01$; ${ }^{* \star} P<0.001$. All statistical analyses were performed using Prism 7 (GraphPad).

\section{Contact for reagent and resource sharing}

Further information for reagents may be directed to, and will be fulfilled by the lead contact, Francesca Sacco (francesca.sacco@ uniroma2.it).

\section{Data and software availability}

The datasets generated and/or analyzed in the current study are available from the corresponding authors on reasonable request.

\section{Supplementary Information}

Supplementary Information is available at https://doi.org/10.26508/lsa. 202000660.

\section{Acknowledgements}

This work was supported by a grant of the European Research Council (grant number 322749 [G Cesareni]) and by Rita Levi Montalcini grant, Ministero dell'Istruzione, Università e Ricerca (MIUR) (F Sacco). This work was partly supported by a grant from the Italian association for cancer research (AIRC) to G Cesareni (Investigator Grant - IG 2013). A Reggio was supported by Fondazione Umberto Veronesi. The authors warmly thank Prof Maria Rosa Ciriolo, from the Department of Biology of the University of Rome Tor Vergata for her kind contribution during the critical evaluation of the manuscript.

\section{Author Contributions}

A Reggio: conceptualization, data curation, formal analysis, investigation, visualization, methodology, and writing-original draft, review, and editing.

M Rosina: conceptualization, data curation, formal analysis, investigation, visualization, methodology, and writing-original draft, review, and editing.

N Krahmer: investigation and methodology.

A Palma: investigation and methodology.

LL Petrilli: investigation.

G Maiolatesi: investigation.

G Massacci: investigation.

I Salvatori: investigation and methodology.

C Valle: resources, investigation, methodology, and writing-review and editing.

S Testa: resources, investigation, methodology, and writing-review and editing.

C Gargioli: investigation, visualization, and methodology.

C Fuoco: investigation, visualization, and methodology.

L Castagnoli: conceptualization, resources, supervision, investigation, visualization, methodology, and writing-review and editing.

G Cesareni: conceptualization, resources, supervision, funding acquisition, visualization, methodology, project administration, and writing-review and editing.

F Sacco: conceptualization, resources, data curation, formal analysis, supervision, funding acquisition, visualization, methodology, project administration, and writing-original draft, review, and editing.

\section{Conflict of Interest Statement}

The authors declare that they have no conflict of interest.

\section{References}

Amit S, Hatzubai A, Birman Y, Andersen JS, Ben-Shushan E, Mann M, BenNeriah Y, Alkalay I (2002) Axin-mediated CKI phosphorylation of betacatenin at Ser 45: A molecular switch for the Wnt pathway. Genes Dev 16: 1066-1076. doi:10.1101/gad.230302

Anderson RM, Barger JL, Edwards MG, Braun KH, O'Connor CE, Prolla TA, Weindruch R (2008) Dynamic regulation of PGC-1alpha localization and turnover implicates mitochondrial adaptation in calorie restriction and the stress response. Aging Cell 7: 101-111. doi:10.1111/ j.1474-9726.2007.00357.x 
Arnold L, Henry A, Poron F, Baba-Amer Y, van Rooijen N, Plonquet A, Gherardi RK, Chazaud B (2007) Inflammatory monocytes recruited after skeletal muscle injury switch into antiinflammatory macrophages to support myogenesis. J Exp Med 204: 1057-1069. doi:10.1084/jem.20070075

Banfi S, D'Antona G, Ruocco C, Meregalli M, Belicchi M, Bella P, Erratico S, Donato E, Rossi F, Bifari F, et al (2018) Supplementation with a selective amino acid formula ameliorates muscular dystrophy in $\mathrm{mdx}$ mice. Sci Rep 8: 14659. doi:10.1038/s41598-018-32613-w

Bansal D, Miyake K, Vogel SS, Groh S, Chen CC, Williamson R, McNeil PL, Campbell KP (2003) Defective membrane repair in dysferlin-deficient muscular dystrophy. Nature 423: 168-172. doi:10.1038/nature01573

Barker RG, Horvath D, van der Poel C, Murphy RM (2017) Benefits of prenatal taurine supplementation in preventing the onset of acute damage in the $\mathrm{mdx}$ mouse. PLoS Curr 9. doi:10.1371/currents.md. 9a3e357a0154d01050b591601cbd4fdb

Brandhorst S, Choi IY, Wei M, Cheng CW, Sedrakyan S, Navarrete G, Dubeau L, Yap LP, Park R, Vinciguerra M, et al (2015) A periodic diet that mimics fasting promotes multi-system regeneration, enhanced cognitive performance, and healthspan. Cell Metab 22: 86-99. doi:10.1016/ j.cmet.2015.05.012

Calderone A, Castagnoli L, Cesareni G (2013) Mentha: A resource for browsing integrated protein-interaction networks. Nat Methods 10: 690-691. doi:10.1038/nmeth.2561

Carpenter AE, Jones TR, Lamprecht MR, Clarke C, Kang IH, Friman O, Guertin DA, Chang JH, Lindquist RA, Moffat J, et al (2006) CellProfiler: Image analysis software for identifying and quantifying cell phenotypes. Genome Biol 7: R100. doi:10.1186/gb-2006-7-10-r100

Cerletti M, Jang YC, Finley LWS, Haigis MC, Wagers AJ (2012) Short-term calorie restriction enhances skeletal muscle stem cell function. Cell Stem Cell 10: 515-519. doi:10.1016/j.stem.2012.04.002

Chen ZL, Shao WJ, Xu F, Liu L, Lin BS, Wei XH, Song ZL, Lu HG, Fantus IG, Weng JP, et al (2015) Acute Wnt pathway activation positively regulates leptin gene expression in mature adipocytes. Cell Signal 27: 587-597. doi:10.1016/j.cellsig.2014.12.012

Christov C, Chretien F, Abou-Khalil R, Bassez G, Vallet G, Authier FJ, Bassaglia Y, Shinin V, Tajbakhsh S, Chazaud B, et al (2007) Muscle satellite cells and endothelial cells: Close neighbors and privileged partners. Mol Biol Cell 18: 1397-1409. doi:10.1091/mbc.E06-08-0693

Civitarese AE, Carling S, Heilbronn LK, Hulver MH, Ukropcova B, Deutsch WA, Smith SR, Ravussin E, CALERIE Pennington Team (2007) Calorie restriction increases muscle mitochondrial biogenesis in healthy humans. PLOS Med 4: e76. doi:10.1371/journal.pmed.0040076

Cox J, Mann M (2008) MaxQuant enables high peptide identification rates, individualized p.p.b.-range mass accuracies and proteome-wide protein quantification. Nat Biotechnol 26: 1367-1372. doi:10.1038/ nbt.1511

Dellavalle A, Maroli G, Covarello D, Azzoni E, Innocenzi A, Perani L, Antonini S, Sambasivan R, Brunelli S, Tajbakhsh S, et al (2011) Pericytes resident in postnatal skeletal muscle differentiate into muscle fibres and generate satellite cells. Nat Commun 2: 499. doi:10.1038/ncomms1508

Emery AE (1998) The muscular dystrophies. BMJ 317: 991-995. doi:10.1007/9781-4939-6430-7_4310.1136/bmj.317.7164.991

Hafner P, Bonati U, Erne B, Schmid M, Rubino D, Pohlman U, Peters T, Rutz E, Frank S, Neuhaus C, et al (2016) Improved muscle function in Duchenne muscular dystrophy through L-arginine and metformin: An investigator-initiated, open-label, single-center, proof-of-conceptstudy. PLoS One 11: e0147634. doi:10.1371/journal.pone.0147634

Han R, Rader EP, Levy JR, Bansal D, Campbell KP (2011) Dystrophin deficiency exacerbates skeletal muscle pathology in dysferlin-null mice. Skelet Muscle 1: 35. doi:10.1186/2044-5040-1-35

Heredia JE, Mukundan L, Chen FM, Mueller AA, Deo RC, Locksley RM, Rando TA, Chawla A (2013) Type 2 innate signals stimulate fibro/adipogenic progenitors to facilitate muscle regeneration. Cell 153: 376-388. doi:10.1016/j.cell.2013.02.053

Hogarth MW, Defour A, Lazarski C, Gallardo E, Manera JD, Partridge TA, Nagaraju K, Jaiswal JK (2019) Fibroadipogenic progenitors are responsible for muscle loss in limb girdle muscular dystrophy 2B. Nat Commun 10: 2430. doi:10.1038/s41467-019-10438-z

Joe AWB, Yi L, Natarajan A, Le Grand F, So L, Wang J, Rudnicki MA, Rossi FMV (2010) Muscle injury activates resident fibro/adipogenic progenitors that facilitate myogenesis. Nat Cell Biol 12: 153-163. doi:10.1038/ ncb2015

Jones AE, Price FD, Le Grand F, Soleimani VD, Dick SA, Megeney LA, Rudnick MA (2015) Wnt/ $\beta$-catenin controls follistatin signalling to regulate satellite cell myogenic potential. Skelet Muscle 5: 14. doi:10.1186/ s13395-015-0038-6

Joseph J, Cho D, Doles J (2018) Metabolomic analyses reveal extensive progenitor cell deficiencies in a mouse model of Duchenne muscular dystrophy. Metabolites 8: 61. doi:10.3390/metabo8040061

Juban G, Saclier M, Yacoub-Youssef H, Kernou A, Arnold L, Boisson C, BenLarbi S, Magnan M, Cuvellier S, Théret M, et al (2018) AMPK activation regulates $L T B P 4$-dependent TGF- $\beta 1$ secretion by pro-inflammatory macrophages and controls fibrosis in Duchenne muscular dystrophy. Cell Rep 25: 2163-2176.e6. doi:10.1016/j.celrep.2018.10.077

Judson RN, Quarta M, Oudhoff MJ, Soliman H, Yi L, Chang CK, Loi G, Vander Werff R, Cait A, Hamer M, et al (2018) Inhibition of methyltransferase Setd7 allows the in vitro expansion of myogenic stem cells with improved therapeutic potential. Cell Stem Cell 22: 177-190.e7. doi:10.1016/j.stem.2017.12.010

Kelstrup CD, Bekker-Jensen DB, Arrey TN, Hogrebe A, Harder A, Olsen JV (2018) Performance evaluation of the $\mathrm{Q}$ exactive HF-X for shotgun proteomics. J Proteome Res 17: 727-738. doi:10.1021/ acs.jproteome.7b00602

Knobloch M, Pilz GA, Ghesquière B, Kovacs WJ, Wegleiter T, Moore DL, Hruzova M, Zamboni N, Carmeliet P, Jessberger S (2017) A fatty acid oxidationdependent metabolic shift regulates adult neural stem cell activity. Cell Rep 20: 2144-2155. doi:10.1016/j.celrep.2017.08.029

Kopinke D, Roberson EC, Reiter JF (2017) Ciliary hedgehog signaling restricts injury-induced adipogenesis. Cell 170: 340-351.e12. doi:10.1016/ j.cell.2017.06.035

Kulak NA, Pichler G, Paron I, Nagaraj N, Mann M (2014) Minimal, encapsulated proteomic-sample processing applied to copy-number estimation in eukaryotic cells. Nat Methods 11: 319-324. doi:10.1038/nmeth.2834

Kunnimalaiyaan S, Schwartz VK, Jackson IA, Clark Gamblin T, Kunnimalaiyaan M (2018) Antiproliferative and apoptotic effect of LY2090314, a GSK-3 inhibitor, in neuroblastoma in vitro. BMC Cancer 18: 560. doi:10.1186/ s12885-018-4474-7

Kuswanto W, Burzyn D, Panduro M, Wang KK, Jang YC, Wagers AJ, Benoist C, Mathis D (2016) Poor repair of skeletal muscle in aging mice reflects a defect in local, interleukin-33-dependent accumulation of regulatory T cells. Immunity 44: 355-367. doi:10.1016/j.immuni.2016.01.009

Latres E, Amini AR, Amini AA, Griffiths J, Martin FJ, Wei Y, Lin HC, Yancopoulos GD, Glass DJ (2005) Insulin-like growth factor-1 (IGF-1) inversely regulates atrophy-induced genes via the phosphatidylinositol 3kinase/Akt/mammalian target of rapamycin (PI3K/Akt/mTOR) pathway. J Biol Chem 280: 2737-2744. doi:10.1074/jbc.M407517200

Lees-Shepard JB, Yamamoto M, Biswas AA, Stoessel SJ, Nicholas SE, Cogswell CA, Devarakonda PM, Schneider MJ, Cummins SM, Legendre NP, et al (2018) Activin-dependent signaling in fibro/adipogenic progenitors causes fibrodysplasia ossificans progressiva. Nat Commun 9: 471. doi:10.1038/s41467-018-02872-2

Lemos DR, Babaeijandaghi F, Low M, Chang CK, Lee ST, Fiore D, Zhang RH, Natarajan A, Nedospasov SA, Rossi FMV (2015) Nilotinib reduces muscle fibrosis in chronic muscle injury by promoting TNF-mediated 
apoptosis of fibro/adipogenic progenitors. Nat Med 21: 786-794 doi:10.1038/nm.3869

Lepper C, Partridge TA, Fan C-M (2011) An absolute requirement for Pax7positive satellite cells in acute injury-induced skeletal muscle regeneration. Development 138: 3639-3646. doi:10.1242/dev.067595

Li W, Zhu C, Li Y, Wu Q, Gao R (2014) Mest attenuates CCl4-induced liver fibrosis in rats by inhibiting the Wnt/ $\beta$-catenin signaling pathway. Gut Liver 8: 282-291. doi:10.5009/gnl.2014.8.3.282

Lindsay A, Chamberlain CM, Witthuhn BA, Lowe DA, Ervasti JM (2018) Dystrophinopathy-associated dysfunction of Krebs cycle metabolism. Hum Mol Genet 71: 304-310. doi:10.1093/hmg/ddy404

Livak KJ, Schmittgen TD (2001) Analysis of relative gene expression data using real-time quantitative PCR and the 2(-Delta Delta C(T)) Method. Methods 25: 402-408. doi:10.1006/meth.2001.1262

Ljubicic V, Jasmin BJ (2015) Metformin increases peroxisome proliferatoractivated receptor $y$ Co-activator- $1 \alpha$ and utrophin a expression in dystrophic skeletal muscle. Muscle Nerve 52: 139-142. doi:10.1002/ mus.24692

Lunt SY, Vander Heiden MG (2011) Aerobic glycolysis: Meeting the metabolic requirements of cell proliferation. Annu Rev Cell Dev Biol 27: 441-464. doi:10.1146/annurev-cellbio-092910-154237

Marinkovic M, Fuoco C, Sacco F, Cerquone Perpetuini A, Giuliani G, Micarelli E, Pavlidou T, Petrilli LL, Reggio A, Riccio F, et al (2019) Fibro-adipogenic progenitors of dystrophic mice are insensitive to NOTCH regulation of adipogenesis. Life Sci Alliance 2: e201900437. doi:10.26508/ Isa.201900437

Mazurek S (2011) Pyruvate kinase type M2: A key regulator of the metabolic budget system in tumor cells. Int J Biochem Cell Biol 43: 969-980. doi:10.1016/j.biocel.2010.02.005

Milad N, White Z, Tehrani AY, Sellers S, Rossi FMV, Bernatchez P (2017) Increased plasma lipid levels exacerbate muscle pathology in the mdx mouse model of Duchenne muscular dystrophy. Skelet Muscle 7: 19. doi:10.1186/s13395-017-0135-9

Mozzetta C, Consalvi S, Saccone V, Tierney M, Diamantini A, Mitchell KJ, Marazzi G, Borsellino G, Battistini L, Sassoon D, et al (2013) Fibroadipogenic progenitors mediate the ability of HDAC inhibitors to promote regeneration in dystrophic muscles of young, but not old Mdx mice. EMBO Mol Med 5: 626-639. doi:10.1002/emmm.201202096

Murphy MM, Lawson JA, Mathew SJ, Hutcheson DA, Kardon G (2011) Satellite cells, connective tissue fibroblasts and their interactions are crucial for muscle regeneration. Development 138: 3625-3637. doi:10.1242/ dev.064162

Ohlendieck K, Campbell KP (1991) Dystrophin-associated proteins are greatly reduced in skeletal muscle from mdx mice. J Cell Biol 115: 1685-1694. doi:10.1083/jcb.115.6.1685

Onopiuk M, Brutkowski W, Wierzbicka K, Wojciechowska S, Szczepanowska J, Fronk J, Lochmüller H, Górecki DC, Zabłocki K (2009) Mutation in dystrophin-encoding gene affects energy metabolism in mouse myoblasts. Biochem Biophys Res Commun 386: 463-466. doi:10.1016/ j.bbrc.2009.06.053

Pala F, Di Girolamo D, Mella S, Yennek S, Chatre L, Ricchetti M, Tajbakhsh S (2018) Distinct metabolic states govern skeletal muscle stem cell fates during prenatal and postnatal myogenesis. I Cell Sci 131: jcs212977. doi:10.1242/jcs.212977

Palamiuc L, Schlagowski A, Ngo ST, Vernay A, Dirrig-Grosch S, Henriques A, Boutillier AL, Zoll J, Echaniz-Laguna A, Loeffler JP, et al (2015) A metabolic switch toward lipid use in glycolytic muscle is an early pathologic event in a mouse model of amyotrophic lateral sclerosis. EMBO Mol Med 7: 526-546. doi:10.15252/emmm.201404433

Passaquin AC, Renard M, Kay L, Challet C, Mokhtarian A, Wallimann T, Ruegg UT (2002) Creatine supplementation reduces skeletal muscle degeneration and enhances mitochondrial function in mdx mice. Neuromuscul Disord 12: 174-182. doi:10.1016/s0960-8966(01)00273-5
Pauly M, Daussin F, Burelle Y, Li T, Godin R, Fauconnier J, Koechlin-Ramonatxo C, Hugon G, Lacampagne A, Coisy-Quivy M, et al (2012) AMPK activation stimulates autophagy and ameliorates muscular dystrophy in the mdx mouse diaphragm. Am J Pathol 181: 583-592. doi:10.1016/ j.ajpath.2012.04.004

Percival JM, Siegel MP, Knowels G, Marcinek DJ (2012) Defects in mitochondrial localization and ATP synthesis in the mdx mouse model of Duchenne muscular dystrophy are not alleviated by PDE5 inhibition. Hum Mol Genet 22: 153-167. doi:10.1093/hmg/dds415

Perfetto L, Briganti L, Calderone A, Perpetuini AC, Iannuccelli M, Langone F, Licata L, Marinkovic M, Mattioni A, Pavlidou T, et al (2015) SIGNOR: A database of causal relationships between biological entities. Nucleic Acids Res. doi:10.1093/nar/gkv1048

Petrof BJ, Shrager JB, Stedman HH, Kelly AM, Sweeney HL (1993) Dystrophin protects the sarcolemma from stresses developed during muscle contraction. Proc Natl Acad Sci U S A 90: 3710-3714. doi:10.1073/ pnas.90.8.3710

Radley-Crabb HG, Fiorotto ML, Grounds MD (2011) The different impact of a high fat diet on dystrophic mdx and control C57Bl/10 mice. PLoS Curr 3: RRN1276. doi:10.1371/currents.RRN1276

Reggio A, Rosina M, Palma A, Cerquone Perpetuini A, Petrilli LL, Gargioli C, Fuoco C, Micarelli E, Giuliani G, Cerretani M, et al (2019a) Adipogenesis of Skeletal Muscle Fibro/Adipogenic Progenitors Is Controlled by the WNT5a/GSK3/ $\beta$-Catenin Axis. Rochester, NY: Social Science Research Network.

Reggio A, Spada F, Rosina M, Massacci G, Zuccotti A, Fuoco C, Gargioli C, Castagnoli L, Cesareni G (2019b) The immunosuppressant drug azathioprine restrains adipogenesis of muscle Fibro/Adipogenic Progenitors from dystrophic mice by affecting AKT signaling. Sci Rep 9: 4360. doi:10.1038/s41598-019-39538-y

Rizzieri DA, Cooley S, Odenike O, Moonan L, Chow KH, Jackson K, Wang X, Brail L, Borthakur G (2016) An open-label phase 2 study of glycogen synthase kinase-3 inhibitor LY2090314 in patients with acute leukemia. Leuk Lymphoma 57: 1800-1806. doi:10.3109/ 10428194.2015 .1122781

Rodriguez-Cruz M, Sanchez R, Escobar RE, Cruz-Guzmán ODR, Lopez-Alarcon M, Bernabe Garcia M, Coral-Vazquez R, Matute G, Velazquez Wong AC (2015) Evidence of insulin resistance and other metabolic alterations in boys with Duchenne or becker muscular dystrophy. Int J Endocrinol 2015: 867273-867278. doi:10.1155/2015/867273

Rudolf A, Schirwis E, Giordani L, Parisi A, Lepper C, Taketo MM, Le Grand F (2016) $\beta$-Catenin activation in muscle progenitor cells regulates tissue repair. Cell Rep 15: 1277-1290. doi:10.1016/j.celrep.2016.04.022

Ryall JG, Dell'Orso S, Derfoul A, Juan A, Zare H, Feng X, Clermont D, Koulnis M, Gutierrez-Cruz G, Fulco M, et al (2015) The NAD(+)-dependent SIRT1 deacetylase translates a metabolic switch into regulatory epigenetics in skeletal muscle stem cells. Cell Stem Cell 16: 171-183. doi:10.1016/ j.stem.2014.12.004

Rybalka E, Timpani CA, Cooke MB, Williams AD, Hayes A (2014) Defects in mitochondrial ATP synthesis in dystrophin-deficient mdx skeletal muscles may be caused by complex I insufficiency. PLoS One 9: e115763. doi:10.1371/journal.pone.0115763

Sacco F, Gherardini PF, Paoluzi S, Saez-Rodriguez J, Helmer-Citterich M, Ragnini-Wilson A, Castagnoli L, Cesareni G (2012) Mapping the human phosphatome on growth pathways. Mol Syst Biol 8: 603. doi:10.1038/ msb.2012.36

Sacco F, Silvestri A, Posca D, Pirrò S, Gherardini PF, Castagnoli L, Mann M, Cesareni G (2016) Deep proteomics of breast cancer cells reveals that metformin rewires signaling networks away from a pro-growth state. Cell Syst 2: 159-171. doi:10.1016/j.cels.2016.02.005

Sambasivan R, Yao R, Kissenpfennig A, Van Wittenberghe L, Paldi A, GayraudMorel B, Guenou H, Malissen B, Tajbakhsh S, Galy A (2011) Pax7- 
expressing satellite cells are indispensable for adult skeletal muscle regeneration. Development 138: 3647-3656. doi:10.1242/dev.067587

Schindelin J, Arganda-Carreras I, Frise E, Kaynig V, Longair M, Pietzsch T, Preibisch S, Rueden C, Saalfeld S, Schmid B, et al (2012) Fiji: An opensource platform for biological-image analysis. Nat Methods 9: 676-682. doi:10.1038/nmeth.2019

Schuh RA, Jackson KC, Khairallah RJ, Ward CW, Spangenburg EE (2012) Measuring mitochondrial respiration in intact single muscle fibers. Am J Physiol Regul Integr Comp Physiol 302: R712-R719. doi:10.1152/ ajpregu.00229.2011

Seale P, Ishibashi J, Scime A, Rudnicki MA (2004) Pax7 is necessary and sufficient for the myogenic specification of CD45+:Sca1+ stem cells from injured muscle. PLoS Biol 2: E130. doi:10.1371/ journal.pbio.0020130

Shannon P, Markiel A, Ozier O, Baliga NS, Wang JT, Ramage D, Amin N, Schwikowski B, Ideker T (2003) Cytoscape: A software environment for integrated models of biomolecular interaction networks. Genome Res 13: 2498-2504. doi:10.1101/gr.1239303

Timpani CA, Hayes A, Rybalka E (2015) Revisiting the dystrophin-ATP connection: How half a century of research still implicates mitochondrial dysfunction in Duchenne Muscular Dystrophy aetiology. Med Hypotheses 85: 1021-1033. doi:10.1016/ j.mehy.2015.08.015

Tyanova S, Temu T, Sinitcyn P, Carlson A, Hein MY, Geiger T, Mann M, Cox J (2016) The Perseus computational platform for comprehensive analysis of (prote)omics data. Nat Methods 13: 731-740. doi:10.1038/ nmeth.3901

Uezumi A, Fukada S-I, Yamamoto N, Takeda S, Tsuchida K (2010) Mesenchymal progenitors distinct from satellite cells contribute to ectopic fat cell formation in skeletal muscle. Nat Cell Biol 12: 143-152. doi:10.1038/ncb2014

Uezumi A, Ito T, Morikawa D, Shimizu N, Yoneda T, Segawa M, Yamaguchi M, Ogawa R, Matev MM, Miyagoe-Suzuki Y, et al (2011) Fibrosis and adipogenesis originate from a common mesenchymal progenitor in skeletal muscle. J Cell Sci 124: 3654-3664. doi:10.1242/jcs.086629

Vallecillo Garcia P, Orgeur M, Vom Hofe-Schneider S, Stumm J, Kappert V, Ibrahim DM, Börno ST, Hayashi S, Relaix F, Hildebrandt K, et al (2017) Odd skipped-related 1 identifies a population of embryonic fibroadipogenic progenitors regulating myogenesis during limb development. Nat Commun 8: 1218. doi:10.1038/s41467-017-01120-3

Voisin V, Sébrié C, Matecki S, Yu H, Gillet B, Ramonatxo M, Israël M, De la Porte $\mathrm{S}$ (2005) L-arginine improves dystrophic phenotype in $\mathrm{mdx}$ mice. Neurobiol Dis 20: 123-130. doi:10.1016/j.nbd.2005.02.010

Wang Z, Li L, Zhao H, Peng S, Zuo Z (2015) Chronic high fat diet induces cardiac hypertrophy and fibrosis in mice. Metabolism 64: 917-925. doi:10.1016/ j.metabol.2015.04.010

Zanzoni A, Montecchi-Palazzi L, Quondam M, Ausiello G, Helmer-Citterich M, Cesareni G (2002) MINT: A molecular INTeraction database. FEBS Lett 513: 135-140. doi:10.1016/s0014-5793(01)03293-8

Zhang J, Chen S, Zhang W, Zhang J, Liu X, Shi H, Che H, Wang W, Li F, Yao L (2008) Human differentiation-related gene NDRG1 is a Myc downstreamregulated gene that is repressed by Myc on the core promoter region. Gene 417: 5-12. doi:10.1016/j.gene.2008.03.002

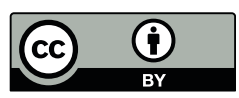

License: This article is available under a Creative Commons License (Attribution 4.0 International, as described at https://creativecommons.org/ licenses/by/4.0/). 